\title{
Improve impact responses of a honeycomb sandwich panel structure with internal resonators
}

\author{
Q.Q. $\mathrm{Li}^{\mathrm{a}}$, Z.C. $\mathrm{He}^{\mathrm{a}, \mathrm{c}}$, E. $\mathrm{Li}^{\mathrm{b}^{*}}$, A.G. Cheng ${ }^{\mathrm{a}}$ \\ ${ }^{\text {a }}$ State Key Laboratory of Advanced Design and Manufacturing for Vehicle Body, Hunan University, \\ Changsha, 410082 P. R. China \\ ${ }^{b}$ School of Science, Engineering \& Design, Teesside University, Middlesbrough, UK \\ ${ }^{c}$ Guangxi Key Laboratory of Automobile Components and Vehicle technology, Guangxi University \\ of Science and Technology, Liuzhou, 545006, P. R. China
}

\begin{abstract}
In this paper, a decahedral honeycomb sandwich panel structure with periodic internal resonators is developed in order to effectively mitigate the impact force and dissipate the impact energy. Five types of local resonance microstructures (LMs) with the property of negative effective mass are applied on the sandwich structure to study the effects of different resonators on load attenuation, and the theoretical and numerical results show that the multi-resonator model (MM) exhibits the best attenuation effect. In addition, the damping characteristic is added to the MM to further improve the structure responses. The analysis results have clearly demonstrated that the combination of damping characteristic and local resonance of multi-resonators can significantly dissipate the impact energy and mitigate the dynamic load. In addition, the optimization analyses are also carried out to optimize the spring stiffness, lumped mass and damping coefficient. The performance of the sandwich panel structure is significantly improved with a relatively small structure mass.
\end{abstract}

Key words: resonator; local resonance microstructure; honeycomb sandwich structure; impact force; impact energy;

\footnotetext{
* Corresponding author.

E-mail address: ericsg2012@gmail.com (E. Li)
} 


\section{Introduction}

Over the past decades, the honeycomb sandwich panel structures are widely used as the reinforcing structure to enhance the energy absorbing in the crash. Many have been conducted to improve the performance of the sandwich panel structures (Hou et al. 2015; Wu, Liu, et al. 2017; Xu, Jiang, and Lee 2017; Yang et al. 2017). example, an explosion test (Dharmasena et al. 2008) was conducted to study the dynamic response of a three-dimensional sandwich panel structure with square honeycomb core composed of austenitic steel alloys. The tests of three-point bending and in-panel compression on one kind of hexahedral aluminum honeycomb sandwich panel (Sun et al. 2017) were performed, and results showed that the collapse mode and structure crashworthiness of the sandwich structure were significantly affected by the structural and adhesive parameters. The mechanical performance of a composite honeycomb sandwich panel was studied through the finite element analysis and three point bending test (Lu et al. 2015), and the failure mode of this panel structure was shown in detail. The mechanical performance of a corrugated core sandwich panel under in-plane compressive load was studied by (Biagi and Bart-Smith 2012), and the failure mechanisms of macro buckling, shear buckling and face wrinkling were identified one by one.

The above studies are mainly focused on the mechanical properties of the sandwich structure and the improvement of the stiffness and the energy absorption efficiency of the sandwich structure. However, the mitigation of impact force is rarely studied. Impact force is a key factor in structural crashworthiness. If the attenuation of the impact force is achieved, the structure can be effectively protected. Moreover, the attenuation of the impact force can also be used to protect the person in the collision, such as the protection of passengers during the crash of vehicles.

In this work, the collision analysis of a decahedral honeycomb sandwich panel structure with internal resonators is carried out to attenuate the impact force. The combination of internal resonators and matrix structures is called the LMs, which can exhibit extraordinary physical properties. These properties include the negative 
effective mass (Li, He, Li, et al. 2018; Li, He, Wang, et al. 2018; Xiao et al. 2019), the negative effective bulk modulus (Liu et al. 2011; Wu, Wang, et al. 2017) and the negative effective shear modulus (Lai et al. 2011; Ying, Lai, and Zhang 2011). Among these properties, the negative effective mass obtained by the LMs can be applied to produce the forbidden bands that prevent the propagation of elastic waves. This bizarre phenomenon is due to the coupling effects of the propagating of waves in the host medium and the resonance motion of oscillators.

Previous scholars have conducted in-depth studies on the factors that affect the performance of LMs. The single-resonator microstructure (Jiao et al. 2015) was applied to realize the negative mass density and mitigate the mechanical wave at the local resonance frequency. Following this, the dual-resonator microstructure (Huang and Sun 2009; Tan, Huang, and Sun 2012) was studied to produce the double band gaps, and the multi-resonator microstructure ( $\mathrm{Li}$ et al. 2019) with more degrees of freedom can generate multiple band gaps to manipulate the mechanical waves better. Consequently, the influences of damping characteristic on the performances of LMs were studied. The wave propagation characteristic in the mass-in-mass damped single-resonator lattice system was studied by (Islam and Newaz 2012; Chen et al. 2016). It was found that the damping can extend the attenuation frequency range of mechanical wave and exhibit the metadamping phenomenon. When the damped oscillators and the beam structure were combined (Thompson 2008), the beam structure can exhibit the mitigation effect of mechanical wave. With high damping coefficients and multiple tuning resonance frequencies, this system can obtain wide regions of attenuation frequency. The global optimization techniques (Bouhlel et al. 2018; Chen et al. 2019; Kadlec and Šeděnka 2018) are also very effective to improve the performance of LMs. The propagation of elastic wave in a beam lattice material characterized by the anti-chiral cell was investigated by (Bacigalupo et al. 2016). The number, location and properties of the oscillators can be designed to expand the width of the band gaps. An in-depth study of LMs composed of rubber-coated inclusions was presented by (Krushynska, Kouznetsova, and Geers 2014). Based on the results of numerical analyses, the optimal microstructure produces the widest frequency band 
gap. The underwater low-frequency sound absorption of plate structure with several layers (Meng et al. 2012) was enhanced through using the genetic algorithm and a nonlinear constrained algorithm. The physical parameters of the plate structure were optimized, and the theoretical and experimental results proved that the optimized plate can realize the broadband absorption of underwater sound.

Besides the above separate studies of resonators and sandwich structures, scholars had attempted to combine resonators and sandwich beam structures together to manipulate the wave propagation. For example, the dissipated multi-resonators were applied in the sandwich beam structure (Chen et al. 2017) to further mitigate and absorb the broadband wave. A new type of sandwich beam with the internal oscillators was shown by (Sharma and Sun 2016), and the system can produce the local resonance and Bragg band gaps simultaneously. A new type of broadband vibration absorbing beam based on multi-frequency vibrator was proposed by (Pai, Hao, and Jiang 2014). Theoretical and numerical analyses showed that the dissipated oscillators produce two band gaps that can be applied to absorb and stop the propagation of waves.

In addition to the above theoretical studies, the engineering applications of resonators with the property of negative effective mass on energy absorption were also studied. The polymer matrix with resonant particles (Wang et al. 2017) was designed to absorb the blast wave energy and attenuate the blast wave. Two composite materials with different geometry parameters were proposed to investigate the attenuation effect, and the energy transfer effect between components was studied by calculating the energy history of the system. The performances of metaconcrete under blast and elastic waves were investigated by (Mitchell, Pandolfi, and Ortiz 2014, 2015). Numerical results showed that the inclusions in metaconcrete can absorb a large amount of wave energy, and reduce the stress on the mortar phase, which greatly improve the resistance of material under dynamic load.

In this work, the impact responses of a decahedral honeycomb sandwich panel structure with internal resonators is studied, and the whole design and optimization process of these resonators are also presented to enhance their attenuation effects. 
First, the performances of five kinds of LMs are studied by means of theoretical and numerical analyses. Then, the effects of damping on the impact responses of the panel structure are studied by adding the dampers on the LMs. The impact force and collision energy are significantly attenuated and dissipated through the coupling of metadamping and local resonance. After that, the non-dominated sorting genetic algorithm II (NSGA-II) (Deb et al. 2002) and the artificial tree (AT) algorithm (Li et al. 2017) are applied to optimize the dissipative multi-resonator model (DMM) to further dissipate more impact energy and reduce the structure mass as well as the peak force.

The paper is organized as follows: Section 2 describes the decahedral honeycomb sandwich panel structure. The main content of Section 3 is about the comparative studies of five kinds of LMs. The combination of local resonance property and metadamping characteristic is studied with the DMM in Section 4, and the results show the significant improvement of the impact responses of the panel structure. Then, the optimization analyses of spring stiffness, lumped mass and damping coefficient are carried out in Section 5. Section 6 presents a summary and outlook on the design and manufacture of LMs. Finally, the conclusions are summarized in Section 7.

\section{The decahedral honeycomb sandwich panel structure}

Fig. 1 shows the decahedral honeycomb sandwich panel structure. From Fig. 1(a), a rigid sphere which mass is $0.21 \mathrm{~kg}$, impacts this structure with initial speed of 64 $\mathrm{km} / \mathrm{h}$, and the whole panel structure is constrained at its both ends. The impact force, collision energy and structure deformation are the output responses. The collision analysis is carried out with commercial software LSDYNA. As shown in Fig. 1(b), this panel structure is composed of a cover plate, a base plate and a decahedral honeycomb structure. The thicknesses of the cover plate, the base plate and the

decahedral honeycomb structure are all $1.5 \mathrm{~mm}$. The material of all components is aluminum alloy 6060-T6, and its effective stress and effective strain curve is shown in Fig. 1(c). The detailed sizes of the panel structure and one decahedral honeycomb cell 
are shown in Fig. 1(d).

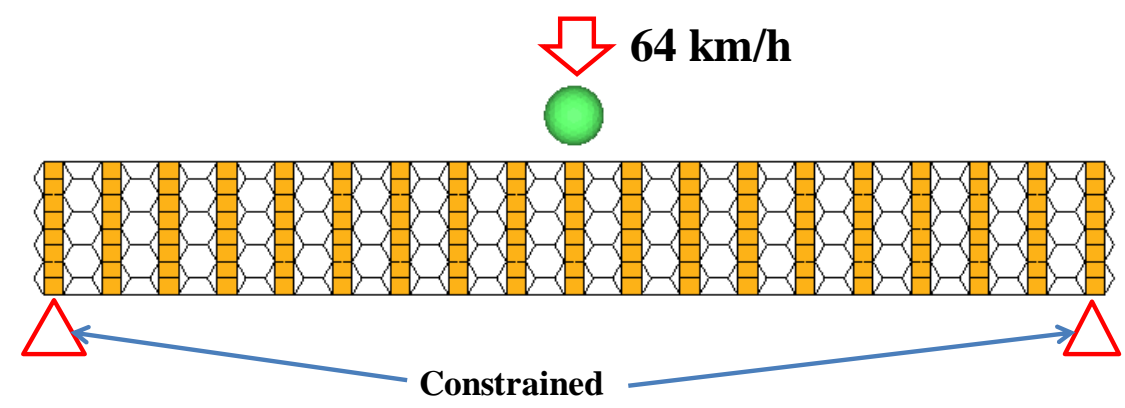

(a)

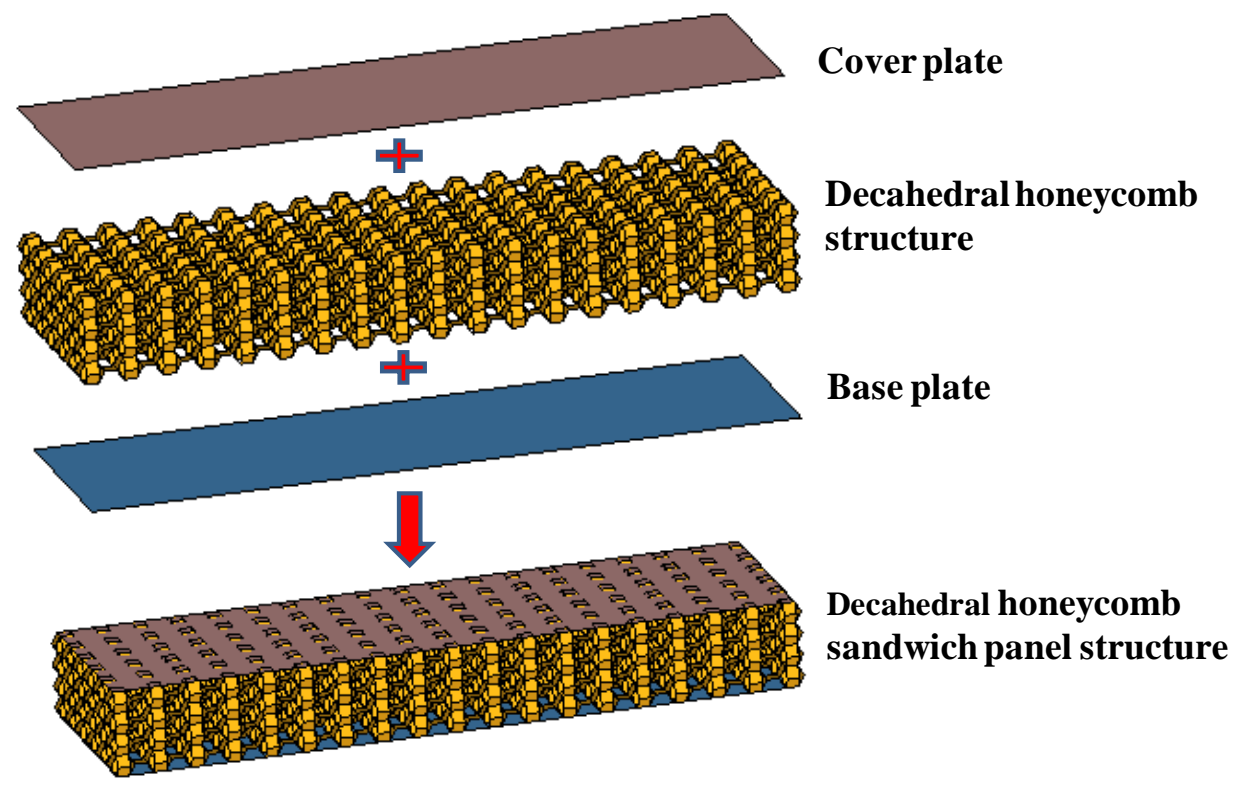

(b)

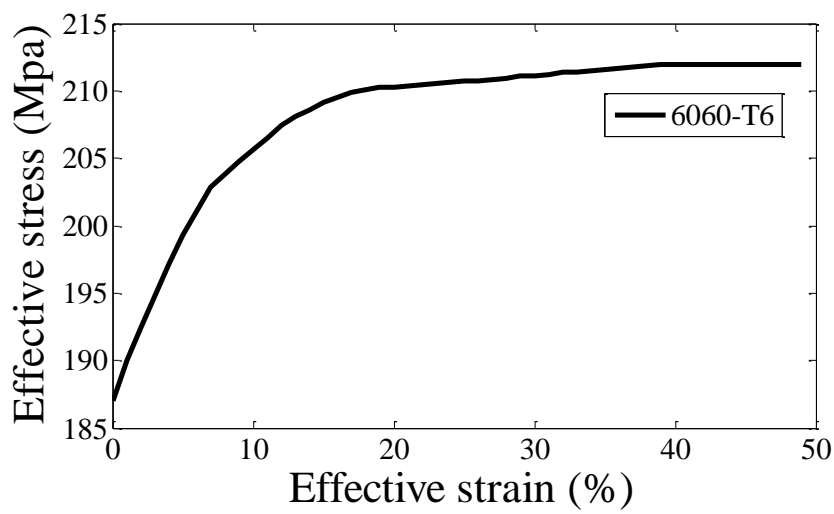

(c)

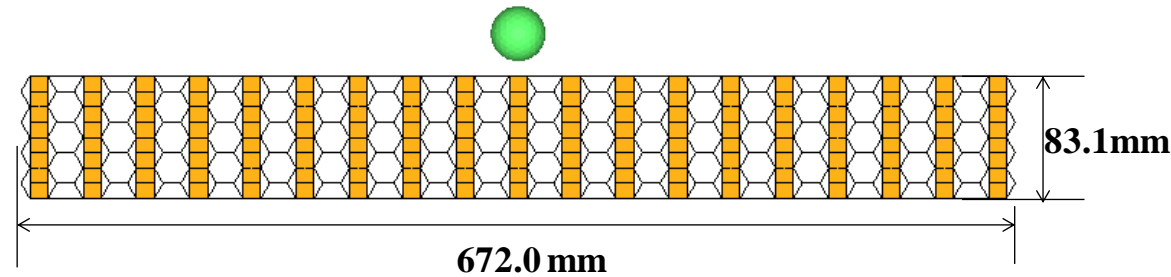



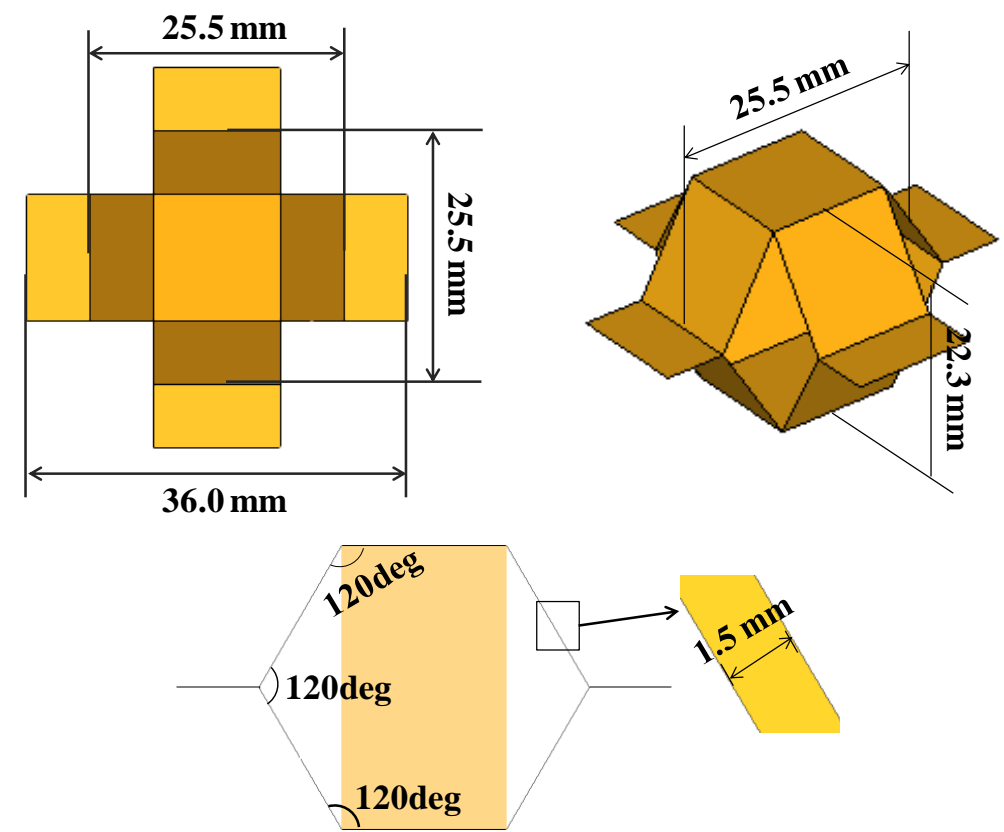

(d)

Fig. 1. (a) The crash model with the three-dimensional sandwich panel structure. (b) The details of the sandwich panel structure. (c) The effective stress and effective strain curve of aluminum alloy 6060-T6. (d) The sizes of the whole impact model and a single decahedral honeycomb cell.

\section{The sandwich panel structure with different internal resonators}

\subsection{Different kinds of LMs}

In this section, the negative mass frequency regions of the physical models of five kinds of LMs are compared to each other, which are the single-resonator, double-resonator, two-resonator, three-resonator and multi-resonator microstructures in Figs. 2(a), (c), (e), (h) and (k).
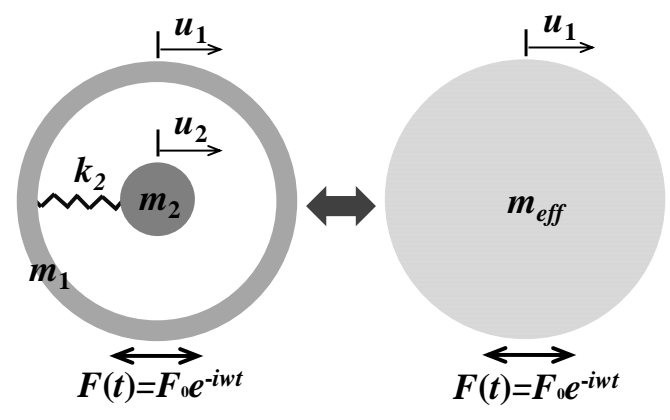

(a)

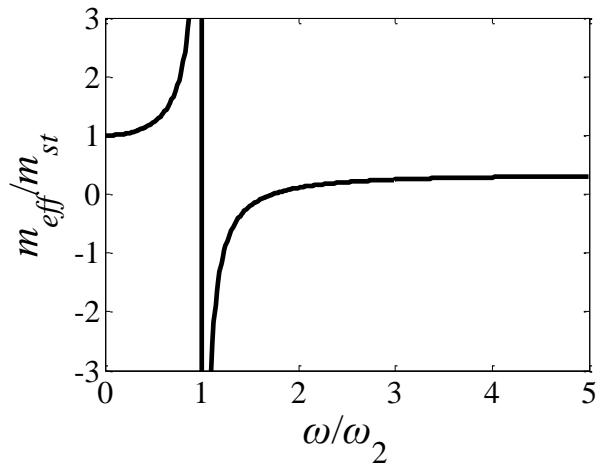

(b) 


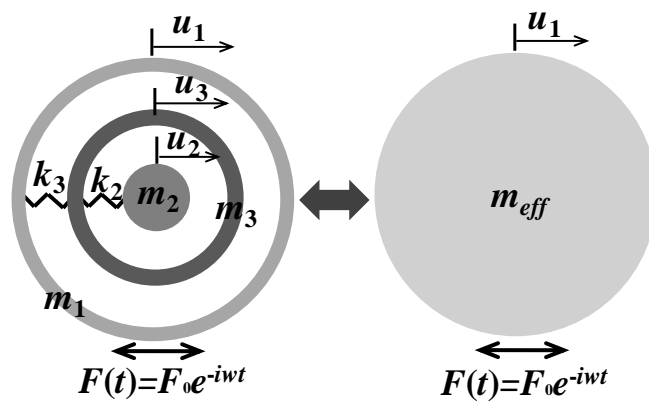

(c)

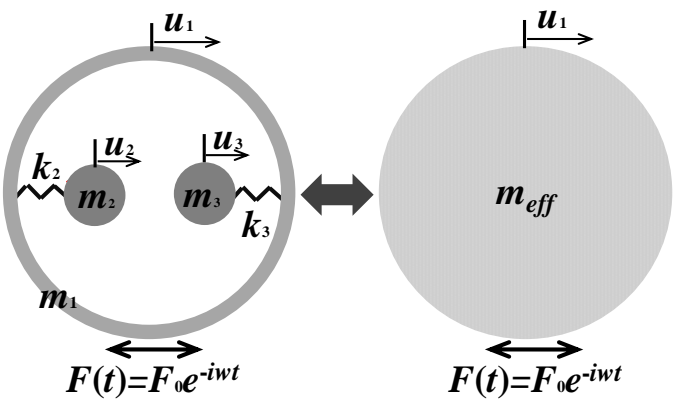

(e)

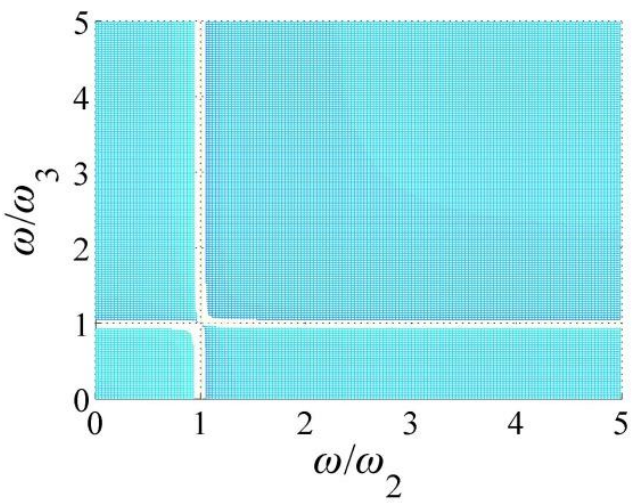

(g)

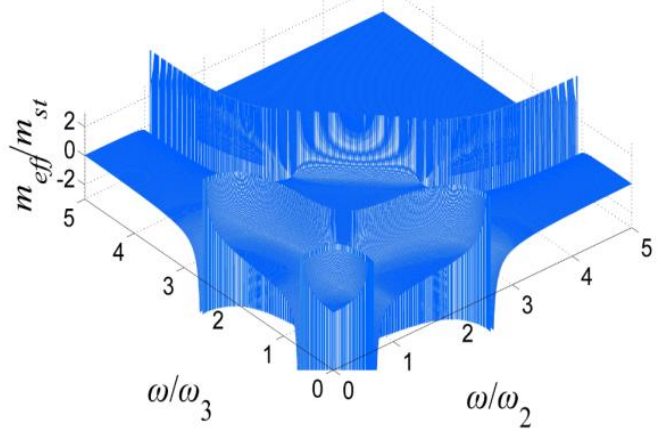

(i)

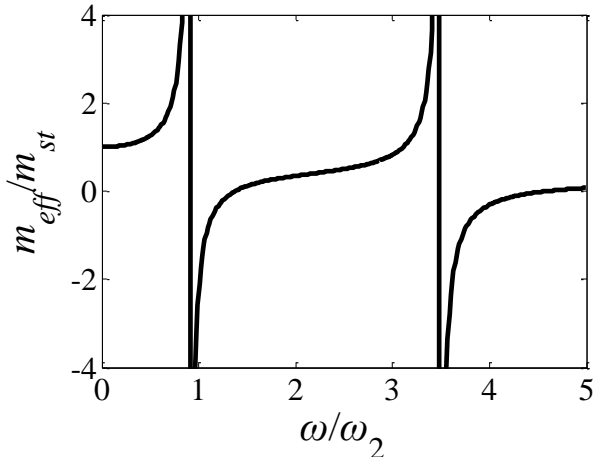

(d)

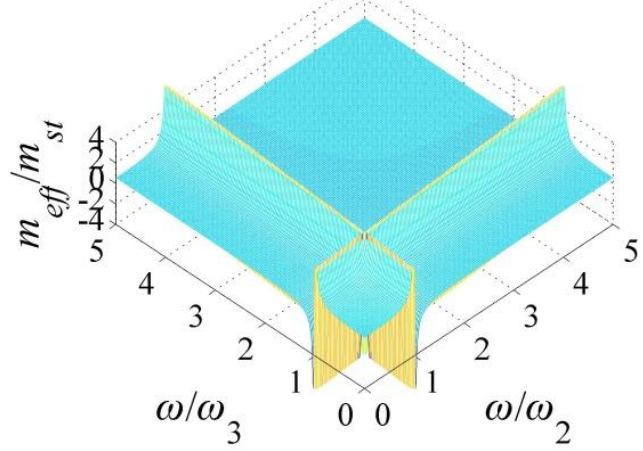

(f)

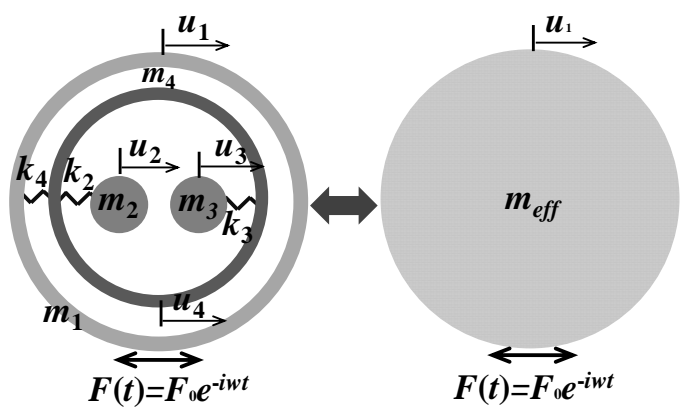

(h)

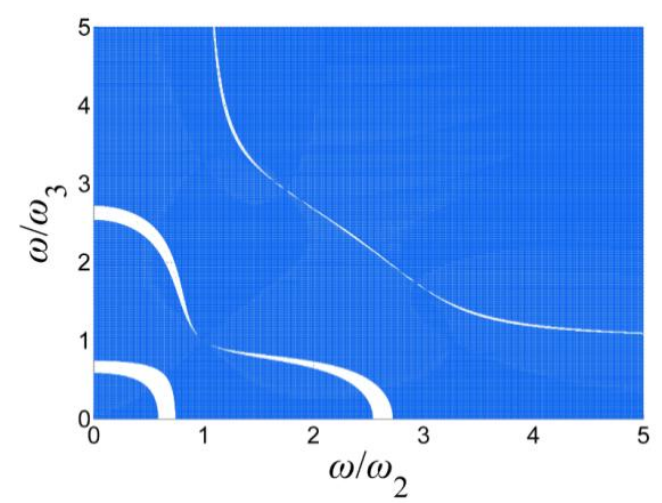

(j) 


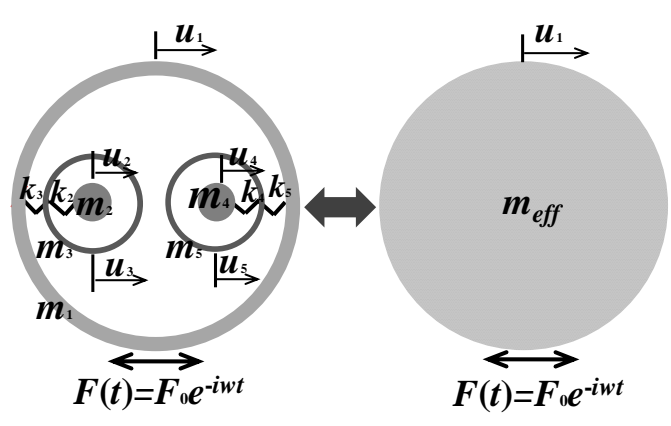

(k)

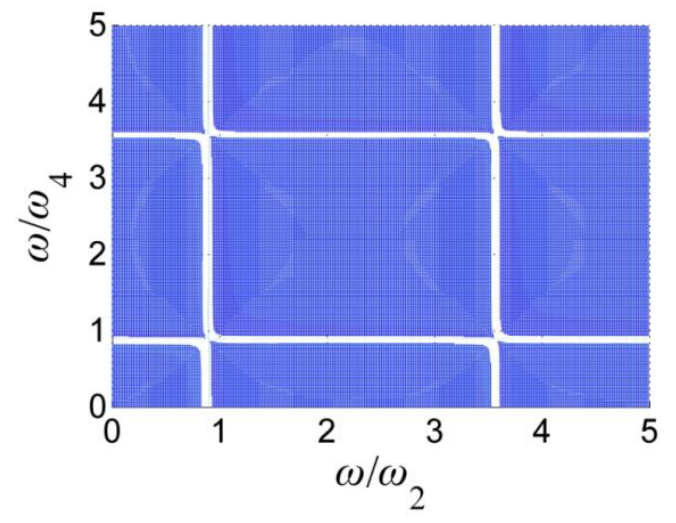

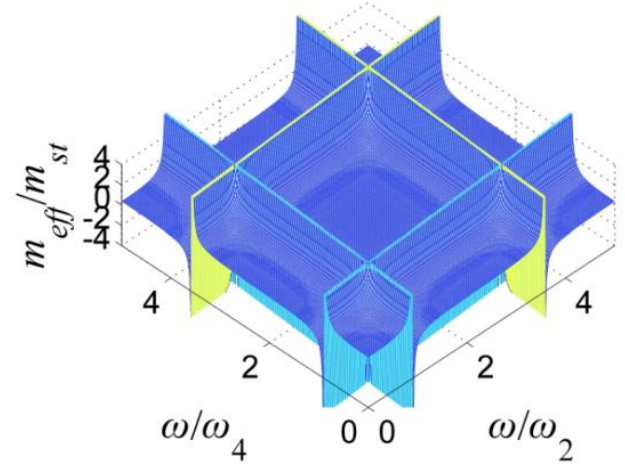

(1)

(m)

Fig. 2. (a) Single-resonator microstructure. (b) Plot of $m_{e f f} / m_{s t}$ against $\omega / \omega_{2}$ of single-resonator microstructure. (c) Double-resonator microstructure. (d) Plot of $m_{\text {eff }} / m_{s t}$ against $\omega / \omega_{2}$ of double-resonator microstructure. (e) Two-resonator microstructure. (f) Surface graph of $m_{\text {eff }} / m_{s t}$ against $\omega / \omega_{2}$ and $\omega / \omega_{3}$ of two-resonator microstructure in global view. (g) Surface graph of two-resonator microstructure in top view. (h) Three-resonator microstructure. (i) Surface graph of $m_{e f f} / m_{s t}$ against $\omega / \omega_{2}$ and $\omega / \omega_{3}$ of three-resonator microstructure in global view. (j) Surface graph of three-resonator microstructure in top view. (k) Multi-resonator microstructure. (l) Surface graph of $m_{\text {eff }} / m_{s t}$ against $\omega / \omega_{2}$ and $\omega / \omega_{4}$ of multi-resonator microstructure in global view. (m) Surface graph of multi-resonator microstructure in top view.

\subsubsection{Single-resonator microstructure}

The single-resonator microstructure which contains two rigid masses is shown in Fig. 2(a). The outer mass is $m_{1}$ which displacement is $u_{1}$. The inner oscillator is $m_{2}$, and its displacement is $u_{2}$. The rigid masses $m_{2}$ and $m_{1}$ are connected by spring $k_{2}$. Force $F$ is applied to the external mass. Based on Newton's Second Law, the motion equations of $m_{1}$ and $m_{2}$ are acquired as Eq. (1). 
$\left[\begin{array}{cc}m_{1} & 0 \\ 0 & m_{2}\end{array}\right]\left\{\begin{array}{l}d^{2} u_{1} / d t^{2} \\ d^{2} u_{2} / d t^{2}\end{array}\right\}+\left[\begin{array}{cc}k_{2} & -k_{2} \\ -k_{2} & k_{2}\end{array}\right]\left\{\begin{array}{l}u_{1} \\ u_{2}\end{array}\right\}=\left\{\begin{array}{l}F \\ 0\end{array}\right\}$

where $t$ is the time. Supposing that the oscillator movements and the outer force follow the simple harmonic behavior, the non-dimensional effective mass can be derived as Eq. (2).

$\frac{m_{e f f}}{m_{s t}}=1+\frac{\theta}{1+\theta}\left[\frac{\left(\omega / \omega_{2}\right)^{2}}{1-\left(\omega / \omega_{2}\right)^{2}}\right]$

where $\theta=m_{2} / m_{1}$ is the ratio of inner mass $m_{2}$ to outer mass $m_{1}$. Fig. 2(b) shows the plot of non-dimensional effective mass $m_{e f f} / m_{s t}$ against non-dimensional frequency $\omega / \omega_{2}$. From Fig. 2(b), a small band gap appears in the resonance frequency, and the effective mass becomes negative in this narrow frequency range.

\subsubsection{Double-resonator microstructure}

Fig. 2(c) illustrates the double-resonator microstructure. There are three lumped masses in this microstructure, which are the external mass $m_{1}$, the middle mass $m_{3}$ and the internal mass $m_{2}$. These rigid masses are connected by springs $k_{2}$ and $k_{3}$. The movements of $m_{1}, m_{3}$ and $m_{2}$ are $u_{1}, u_{3}$ and $u_{2}$. The motion equations of these oscillators are obtained as Eq. (3).

$$
\left[\begin{array}{ccc}
m_{1} & 0 & 0 \\
0 & m_{2} & 0 \\
0 & 0 & m_{3}
\end{array}\right]\left\{\begin{array}{l}
d^{2} u_{1} / d t^{2} \\
d^{2} u_{2} / d t^{2} \\
d^{2} u_{3} / d t^{2}
\end{array}\right\}+\left[\begin{array}{ccc}
k_{3} & 0 & -k_{3} \\
0 & k_{2} & -k_{2} \\
-k_{3} & -k_{2} & k_{2}+k_{3}
\end{array}\right]\left\{\begin{array}{l}
u_{1} \\
u_{2} \\
u_{3}
\end{array}\right\}=\left\{\begin{array}{l}
F \\
0 \\
0
\end{array}\right\}
$$

Based on Eq. (3), the non-dimensional effective mass $m_{e f f} / m_{s t}$ of this double-resonator microstructure can be calculated as Eq. (4).

$$
\frac{m_{e f f}}{m_{s t}}=\frac{\theta_{1}}{\theta_{1}+\theta_{3}+\theta_{1} \theta_{3}}+\frac{\theta_{3}}{\theta_{1}+\theta_{3}+\theta_{1} \theta_{3}}\left[\frac{1+\theta_{1}-\left(\omega / \omega_{2}\right)^{2}}{\left[1-\left(\omega / \omega_{2}\right)^{2}\right]\left[1+\delta_{1}-\left(\delta_{1} / \theta_{1}\right)\left(\omega / \omega_{2}\right)^{2}\right]-\delta_{1}}\right]
$$

where the mass ratios are $\theta_{1}=m_{2} / m_{3}$ and $\theta_{3}=m_{2} / m_{1}$, the stiffness ratio is $\delta_{1}=k_{2} / k_{3}$, the local resonance frequency of the inner mass $m_{2}$ is $\omega_{2}=\sqrt{k_{2} / m_{2}}$. Fig. 2(d) shows the plot of $m_{\text {eff }} / m_{s t}$ against $\omega / \omega_{2}$. There are two band gaps in Fig. 2(d), and the non-dimensional effective mass is negative in these frequency regions. The width of these two band gaps is larger than that of the single band gap, which means the 
double-resonator microstructure can better manipulate the mechanical waves compared with the single-resonator microstructure.

\subsubsection{Two-resonator microstructure}

Fig. 2(e) illustrates the two-resonator microstructure which also has three lumped masses $m_{1}, m_{2}$ and $m_{3}$. The displacement of each mass is $u_{1}, u_{2}$ and $u_{3}$. The springs $k_{2}$ and $k_{3}$ connect $m_{1}, m_{2}$ and $m_{3}$. The motion equations of these masses are shown as follows.

$$
\left[\begin{array}{ccc}
m_{1} & 0 & 0 \\
0 & m_{2} & 0 \\
0 & 0 & m_{3}
\end{array}\right]\left\{\begin{array}{l}
d^{2} u_{1} / d t^{2} \\
d^{2} u_{2} / d t^{2} \\
d^{2} u_{3} / d t^{2}
\end{array}\right\}+\left[\begin{array}{ccc}
k_{2}+k_{3} & -k_{2} & -k_{3} \\
-k_{2} & k_{2} & 0 \\
k_{3} & 0 & -k_{3}
\end{array}\right]\left\{\begin{array}{l}
u_{1} \\
u_{2} \\
u_{3}
\end{array}\right\}=\left\{\begin{array}{l}
F \\
0 \\
0
\end{array}\right\}
$$

Analogous to the single-resonator and double-resonator microstructures, the non-dimensional effective mass $m_{e f f} m_{s t}$ of this model is acquired as Eq. (6).

$$
\frac{m_{e f f}}{m_{s t}}=\frac{1}{1+\theta_{2}+\theta_{3}}+\frac{1}{1+\theta_{2}+\theta_{3}}\left[\theta_{2} \frac{1}{1-\left(\omega / \omega_{2}\right)^{2}}+\theta_{3} \frac{1}{1-\left(\omega / \omega_{3}\right)^{2}}\right]
$$

where the mass ratios are $\theta_{2}=m_{2} / m_{1}$ and $\theta_{3}=m_{3} / m_{1}$. Surface graphs of $m_{\text {eff }} / m_{s t}$ against $\omega / \omega_{2}$ and $\omega / \omega_{3}$ in global and top views are shown in Figs. 2(f) and (g). Two orthogonal band gaps appear in these surface plots. When $\omega / \omega_{2}$ and $\omega / \omega_{3}$ approach 1 , two band gaps are generated on the $\omega / \omega_{2}$ and $\omega / \omega_{3}$ axes, respectively. Differ from the double-resonator microstructure, there is no coupling between these two band gaps, which is convenient for us to design the two band gaps separately to mitigate the critical spectrum areas.

\subsubsection{Three-resonator microstructure}

The three-resonator microstructure is illustrated in Fig. 2(h). There are four rigid masses $m_{1}, m_{2}, m_{3}$ and $m_{4}$ in this microstructure, and their displacements are $u_{1}, u_{2}, u_{3}$ and $u_{4}$. The spring $k_{2}$ connects lumped masses $m_{2}$ and $m_{4}$, the spring $k_{3}$ connects resonators $m_{3}$ and $m_{4}$, and the spring $k_{4}$ connects oscillators $m_{1}$ and $m_{4}$. The motion equations of these resonators can be written as follows. 


$$
\left[\begin{array}{cccc}
m_{1} & 0 & 0 & 0 \\
0 & m_{2} & 0 & 0 \\
0 & 0 & m_{3} & 0 \\
0 & 0 & 0 & m_{4}
\end{array}\right]\left\{\begin{array}{l}
d^{2} u_{1} / d t^{2} \\
d^{2} u_{2} / d t^{2} \\
d^{2} u_{3} / d t^{2} \\
d^{2} u_{4} / d t^{2}
\end{array}\right\}+\left[\begin{array}{cccc}
k_{4} & 0 & 0 & -k_{4} \\
0 & k_{2} & 0 & -k_{4} \\
0 & 0 & k_{3} & -k_{3} \\
-k_{4} & -k_{2} & -k_{3} & k_{2}+k_{3}+k_{4}
\end{array}\right]\left\{\begin{array}{l}
u_{1} \\
u_{2} \\
u_{3} \\
u_{4}
\end{array}\right\}=\left\{\begin{array}{l}
F \\
0 \\
0 \\
0
\end{array}\right\}
$$

Then, the effective mass $m_{\text {eff }}$ of this three-resonator microstructure can be obtained as Eq. (8).

$$
m_{e f f}=m_{1}+m_{4} H
$$

where $H$ represents $\frac{1+\frac{\theta_{2}}{1-\left(\omega / \omega_{2}\right)^{2}}+\frac{\theta_{3}}{1-\left(\omega / \omega_{3}\right)^{2}}}{1-\frac{\delta_{2}\left(\omega / \omega_{2}\right)^{2}}{2 \theta_{2}}-\frac{\delta_{3}\left(\omega / \omega_{3}\right)^{2}}{2 \theta_{3}}-\frac{\delta_{2}\left(\omega / \omega_{2}\right)^{2}}{1-\left(\omega / \omega_{2}\right)^{2}}-\frac{\delta_{3}\left(\omega / \omega_{3}\right)^{2}}{1-\left(\omega / \omega_{3}\right)^{2}}}$ The stiffness ratios are $\delta_{2}=k_{2} / k_{4}$ and $\delta_{3}=k_{3} / k_{4}$. The mass ratios are $\theta_{2}=m_{2} / m_{4}$ and $\theta_{3}=$ $m_{3} / m_{4}$. Finally, the non-dimensional effective mass $m_{\text {eff }} / m_{s t}$ of this new microstructure can be acquired as Eq. (9).

$$
\begin{aligned}
\frac{m_{e f f}}{m_{s t}}= & \frac{1}{1+\theta_{2} \gamma_{4}+\theta_{3} \gamma_{4}+\gamma_{4}} \\
& +\frac{\gamma_{4}}{1+\theta_{2} \gamma_{4}+\theta_{3} \gamma_{4}+\gamma_{4}} \frac{1+\frac{\theta_{2}}{1-\left(\omega / \omega_{2}\right)^{2}}+\frac{\theta_{3}}{1-\left(\omega / \omega_{3}\right)^{2}}}{2-\frac{\delta_{2}\left(\omega / \omega_{2}\right)^{2}}{2 \theta_{2}}-\frac{\delta_{3}\left(\omega / \omega_{3}\right)^{2}}{2 \theta_{3}}-\frac{\delta_{2}\left(\omega / \omega_{2}\right)^{2}}{1-\left(\omega / \omega_{2}\right)^{2}}-\frac{\delta_{3}\left(\omega / \omega_{3}\right)^{2}}{1-\left(\omega / \omega_{3}\right)^{2}}}
\end{aligned}
$$

where $\gamma_{4}=m_{4} / m_{1}$ is the ratio of middle mass to outer mass.

Figs. 2(i) and (j) show the surface graphs of $m_{e f f} / m_{s t}$ against $\omega / \omega_{2}$ and $\omega / \omega_{3}$ in global and top views. Three band gaps appear in these surface graphs, and these band gaps are symmetric along the center line of $\omega / \omega_{2}$ and $\omega / \omega_{3}$ axes. These large negative effective mass frequency regions imply its great performance in manipulate mechanical waves.

\subsubsection{Multi-resonator microstructure}

As shown in Fig. 2(k), the multi-resonator microstructure is composed of five rigid masses $m_{1}, m_{2}, m_{3}, m_{4}$ and $m_{5}$. The movements of these oscillators are $u_{1}, u_{2}, u_{3}$, $u_{4}$ and $u_{5}$, respectively, and the spring which connects these resonators are $k_{1}, k_{2}, k_{3}$ and $k_{4}$. Therefore, the control equation of the multi-resonator microstructure is 
summarized as follows.

$$
\begin{aligned}
{\left[\begin{array}{ccccc}
m_{1} & 0 & 0 & 0 & 0 \\
0 & m_{2} & 0 & 0 & 0 \\
0 & 0 & m_{3} & 0 & 0 \\
0 & 0 & 0 & m_{4} & 0 \\
0 & 0 & 0 & 0 & m_{5}
\end{array}\right]\left\{\begin{array}{l}
d^{2} u_{1} / d t^{2} \\
d^{2} u_{2} / d t^{2} \\
d^{2} u_{3} / d t^{2} \\
d^{2} u_{4} / d t^{2} \\
d^{2} u_{5} / d t^{2}
\end{array}\right\}+} \\
{\left[\begin{array}{ccccc}
k_{3}+k_{5} & 0 & -k_{3} & 0 & -k_{5} \\
0 & k_{2} & -k_{2} & 0 & 0 \\
-k_{3} & -k_{2} & k_{2}+k_{3} & 0 & 0 \\
0 & 0 & 0 & k_{4} & -k_{4} \\
-k_{5} & 0 & 0 & -k_{4} & k_{4}+k_{5}
\end{array}\right]\left\{\begin{array}{l}
u_{1} \\
u_{2} \\
u_{3} \\
u_{4} \\
u_{5}
\end{array}\right\}=\left\{\begin{array}{l}
F \\
0 \\
0 \\
0 \\
0
\end{array}\right\} }
\end{aligned}
$$

Then, the non-dimensional effective mass $m_{\text {eff }} / m_{s t}$ of this multi-resonator microstructure can be obtained as Eq. (11).

$$
\begin{aligned}
\frac{m_{e f f}}{m_{s t}} & =\frac{1}{1+\left(\theta_{2}+1\right) \gamma_{3}+\left(1+\theta_{4}\right) \gamma_{5}} \\
& +\frac{\gamma_{3}}{1+\left(\theta_{2}+1\right) \gamma_{3}+\left(1+\theta_{4}\right) \gamma_{5}} \frac{\left(\left(\omega / \omega_{2}\right)^{2}-\theta_{2}-1\right) \theta_{2}}{\delta_{2}\left(\omega / \omega_{2}\right)^{2}\left(\theta_{2}-\left(\omega / \omega_{2}\right)^{2}+1\right)+\theta_{2}\left(\left(\omega / \omega_{2}\right)^{2}-1\right)} \\
& +\frac{\gamma_{5}}{1+\left(\theta_{2}+1\right) \gamma_{3}+\left(1+\theta_{4}\right) \gamma_{5}} \frac{\left(\left(\omega / \omega_{4}\right)^{2}-\theta_{4}-1\right) \theta_{4}}{\delta_{4}\left(\omega / \omega_{4}\right)^{2}\left(\theta_{4}-\left(\omega / \omega_{4}\right)^{2}+1\right)+\theta_{4}\left(\left(\omega / \omega_{4}\right)^{2}-1\right)}
\end{aligned}
$$

where the mass ratios are $\theta_{2}=m_{2} / m_{3}, \gamma_{3}=m_{3} / m_{1}, \theta_{4}=m_{4} / m_{5}$ and $\gamma_{5}=m_{5} / m_{1}$, the stiffness ratios are $\delta_{2}=k_{2} / k_{3}$ and $\delta_{4}=k_{4} / k_{5}$. Figs. 2(l) and (m) are the surface graphs of $m_{\text {eff }} / m_{s t}$ against $\omega / \omega_{2}$ and $\omega / \omega_{4}$ in global and top views. There are four band gaps in Figs. 2(l) and (m), two of these band gaps appear on the $\omega / \omega_{2}$ axis and the other two appear on the $\omega / \omega_{4}$ axis. Furthermore, these multiple band gaps mean wide negative mass frequency regions and great design freedom for spectrum attenuation.

\subsection{Impact analyses of different resonator models}

As shown in Fig. 3, an oscillator is applied in one decahedral honeycomb cell, and the combination of one oscillator and one decahedral honeycomb cell constitutes a single-resonator microstructure. The decahedral honeycomb cell represents the outer mass $m_{1}$ of the microstructure. Based on the same principle, the other four types of oscillators are also applied in this sandwich panel structure, respectively. With this combination of the resonators and the decahedral honeycomb cells, this sandwich 
panel structure has the ability to mitigate the impact force. Then, the attenuation effects of the panel structure with these five kinds of resonators are compared with each other by studying their impact results. The oscillator masses of all LMs are 0.005 $\mathrm{kg}$, and the spring stiffness of each microstructure is shown in Table 1.

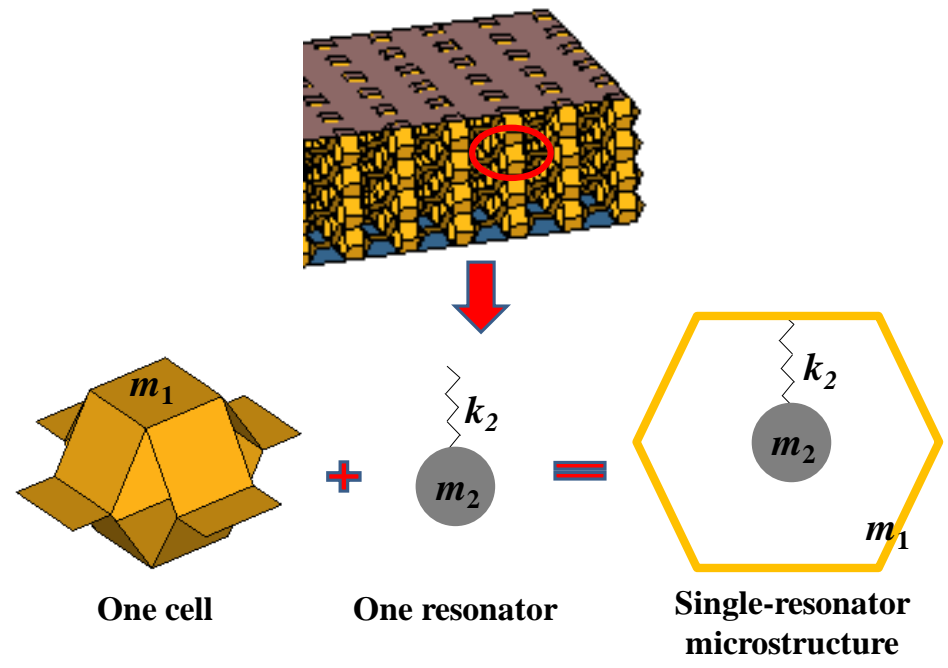

Fig. 3. The panel structure with single-resonator.

Table 1. The spring stiffness of these five kinds of LMs

\begin{tabular}{cc}
\hline & Spring stiffness $(\mathrm{N} / \mathrm{mm})$ \\
\hline Single-resonator & $k_{2}=7.9$ \\
Double-resonator & $k_{2}=17.7, k_{3}=7.9$ \\
Two-resonator & $k_{2}=17.7, k_{3}=7.9$ \\
Three-resonator & $k_{2}=31.6, k_{3}=17.7, k_{4}=7.9$ \\
Multi-resonator & $k_{2}=31.6, k_{3}=1.8, k_{4}=17.7, k_{5}=7.9$ \\
\hline
\end{tabular}

The results of impact forces of these models are shown in Figs. 4, 5 and Table 2. The original model is the sandwich structure without any oscillator, and its peak impact force is $23.9 \mathrm{kN}$. The peak forces of the sandwich panel structure with single-resonator, double-resonator, two-resonator, three-resonator and multi-resonator cores are $22.1 \mathrm{kN}, 19.9 \mathrm{kN}, 19.2 \mathrm{kN}, 17.7 \mathrm{kN}$ and $15.3 \mathrm{kN}$, respectively. Obviously, the impact force is significantly attenuated with these LMs. Furthermore, with the same unit cell number, the attenuation effect of the multi-resonator is the best among these models.

From Figs. 4(a) and (b), the impact force, kinetic energy and internal energy curves of original model keep oscillating with time. The initial kinetic energy and 
initial internal energy are $34.16 \mathrm{~J}$ and $0 \mathrm{~J}$. These imply the absorption efficiency of impact energy is low. In addition, Fig. 4 (c) shows the deformation nephogram of the sandwich panel structure at different time. It can be seen that when $t=2 \mathrm{~ms}$, the deformation is mainly concentrated in the middle area of the structure, and the maximum deformation is $4.64 \mathrm{~mm}$. When $t=5 \mathrm{~ms}$, the deformation zone is enlarged, and its peak deformation is $6.58 \mathrm{~mm}$. Furthermore, when the time is increased to $10 \mathrm{~ms}, 15 \mathrm{~ms}$ or $20 \mathrm{~ms}$, the deformation region of the structure is still very large, and the maximum deformations are $4.51 \mathrm{~mm}, 6.05 \mathrm{~mm}$ and 6.20 , respectively. Therefore, the deformation region of the structure is large, and the deformation does not decrease with time.

From Fig. 5, for these resonator models, their amplitudes of impact force, kinetic energy and internal energy curves are reduced compared with the original model, and the decline of the MM is the most pronounced. These mean that much impact energy is stored in the MM. Therefore, based on the best performances of impact force mitigation and impact energy storage, the multi-resonator is selected for next study.

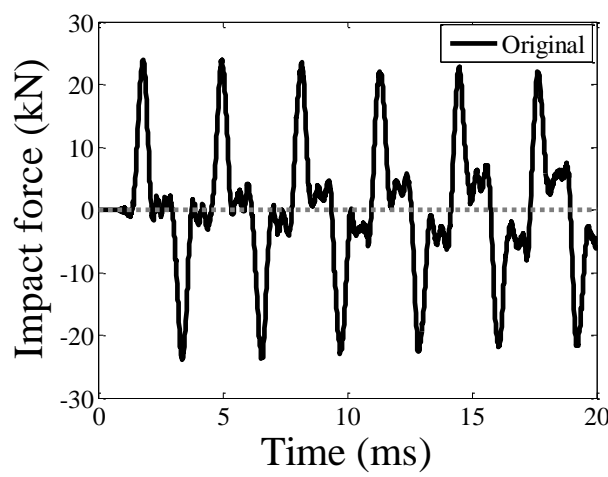

(a)

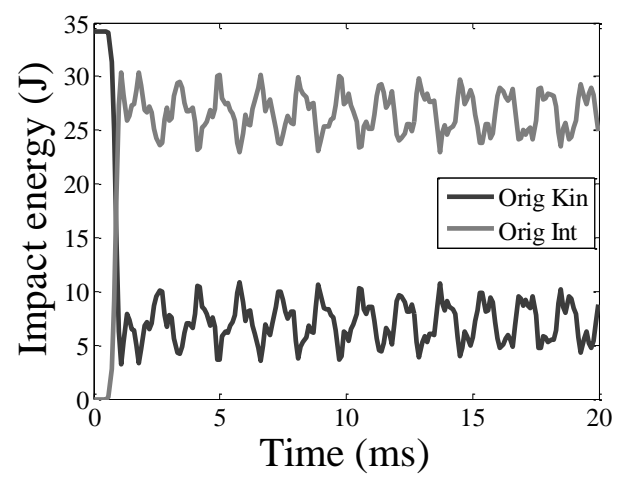

(b) 

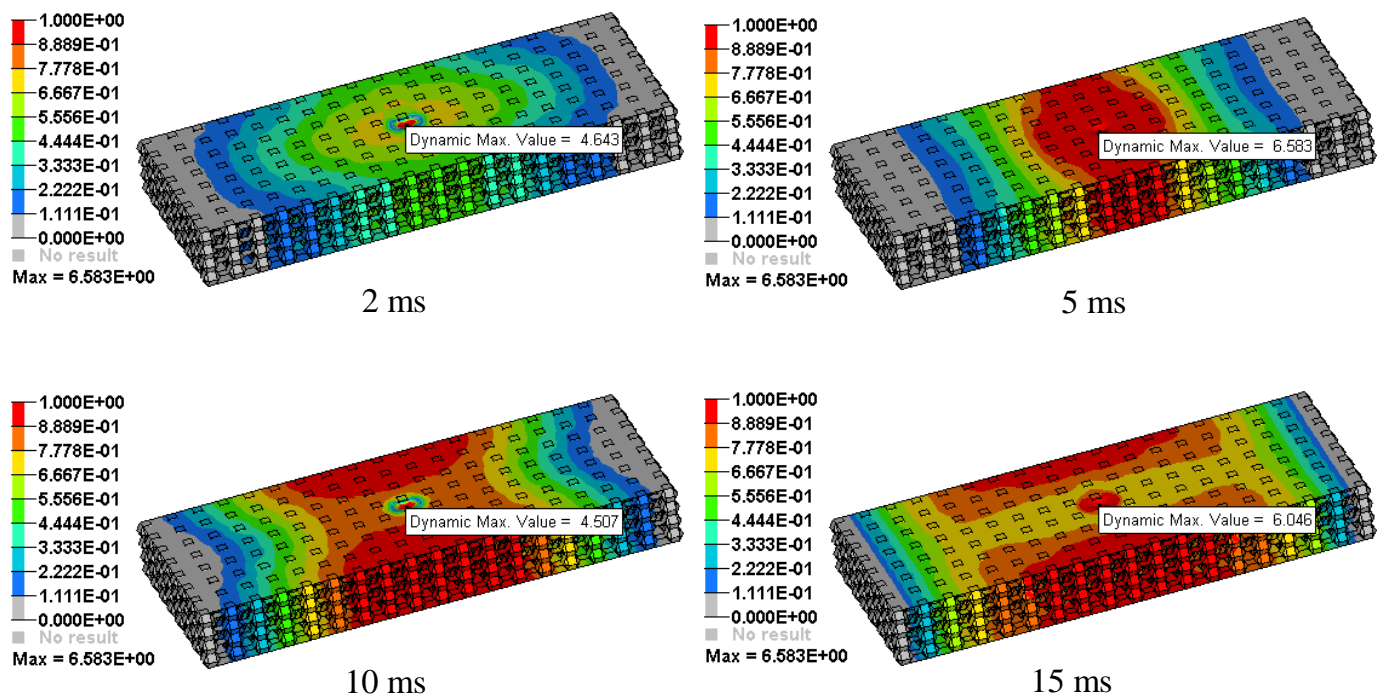

$15 \mathrm{~ms}$

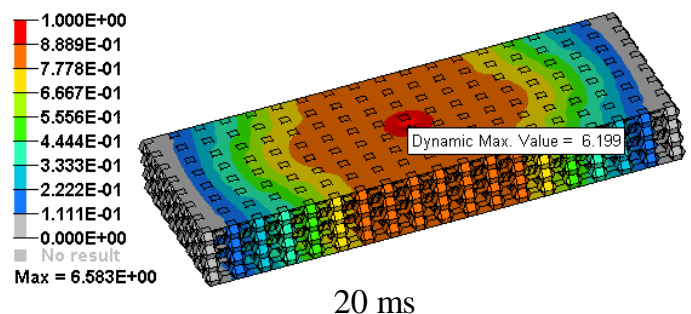

(c)

Fig. 4. The impact results of the original model of (a) impact force curve, (b) kinetic (Kin) energy and internal (Int) energy curves, (c) deformation nephogram.
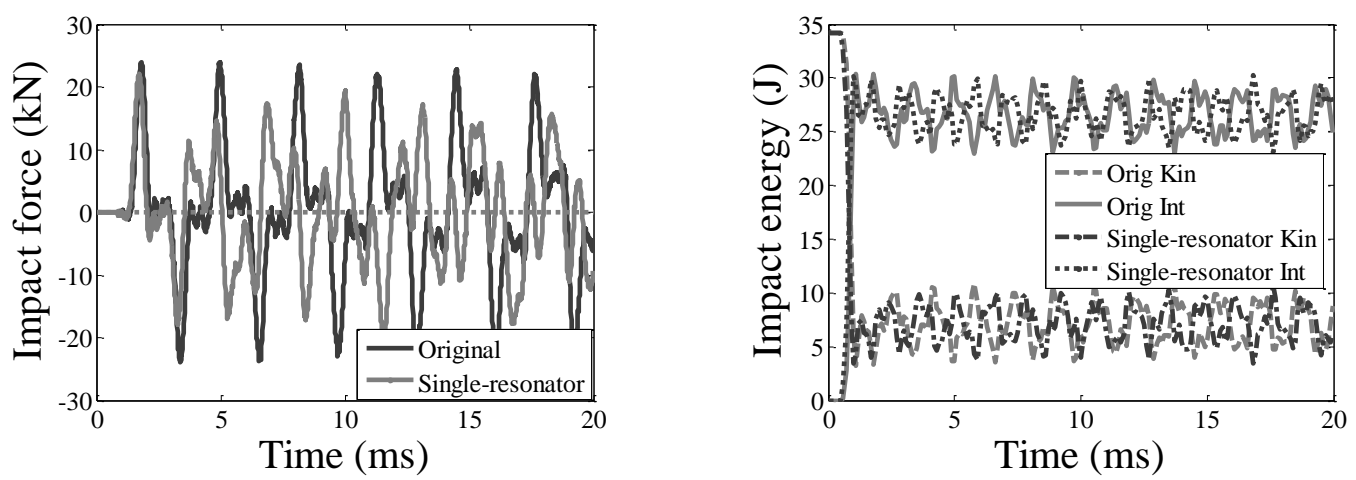

(a) 

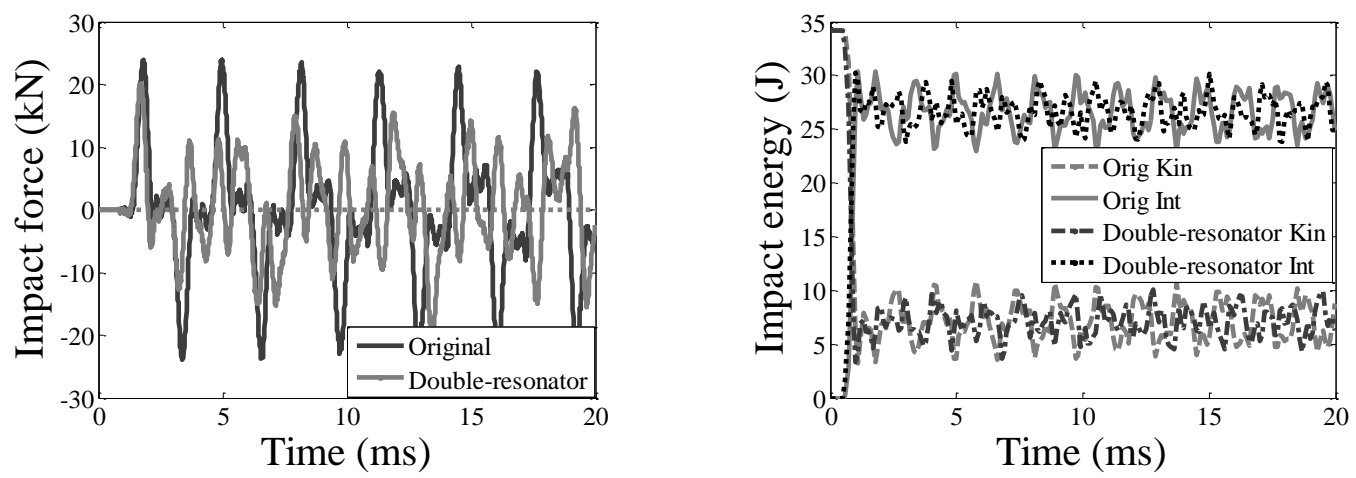

(b)
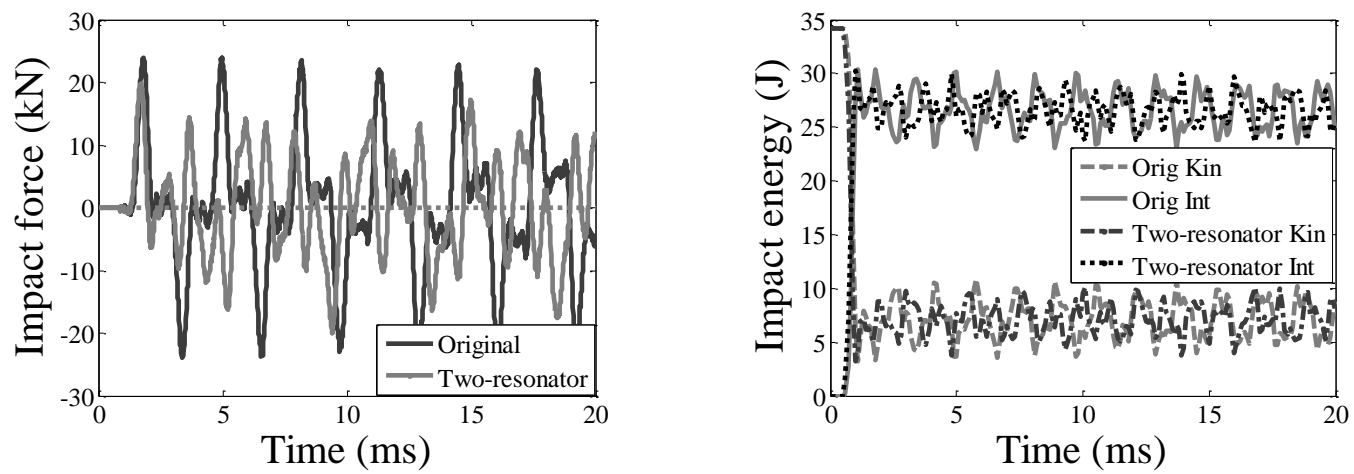

(c)
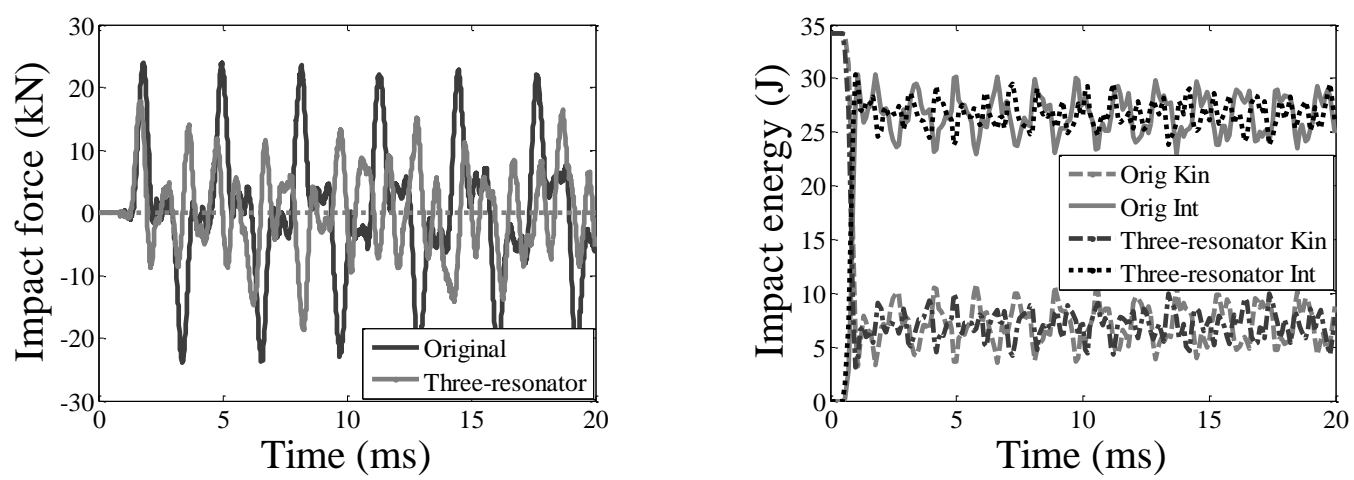

(d)
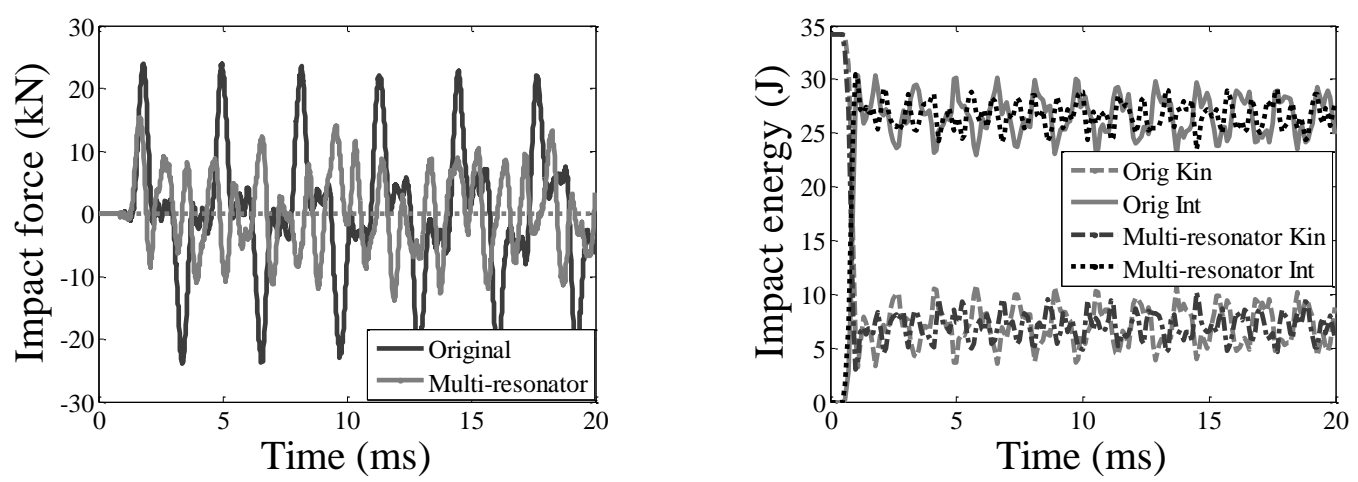

(e) 
Fig. 5. Impact force and impact energy curves of the (a) single-resonator, (b) double-resonator, (c) two-resonator, (d) three-resonator, and (e) multi-resonator models.

Table 2. The impact results these five kinds of resonator models

\begin{tabular}{cc}
\hline & Peak impact force $(\mathrm{kN})$ \\
\hline Original & 23.9 \\
Single-resonator & 22.1 \\
Double-resonator & 19.9 \\
Two-resonator & 19.9 \\
Three-resonator & 17.7 \\
Multi-resonator & 15.3 \\
\hline
\end{tabular}

\section{Sandwich panel structure with dissipative multi-resonators}

\subsection{Dissipative multi-resonator microstructure}

In this section, the effect of damping characteristic on the performance of $\mathrm{MM}$ is studied, and the damper is applied on the MM. Fig. 6(a) illustrates the dissipative multi-resonator microstructure. This structure is composed of the multi-resonator microstructure and four dashpots with damping coefficient $c_{2}, c_{3}, c_{4}$ and $c_{5}$ in parallel to the spring $k_{2}, k_{3}, k_{4}$ and $k_{5}$. The control equations of this microstructure are obtained as Eq. (12).

$$
\begin{aligned}
& m_{2} \frac{d^{2} u_{2}}{d t^{2}}=k_{2}\left(u_{3}-u_{2}\right)+c_{2}\left(\frac{d u_{3}}{d t}-\frac{d u_{2}}{d t}\right) \\
& m_{3} \frac{d^{2} u_{3}}{d t^{2}}=k_{2}\left(u_{2}-u_{3}\right)+k_{3}\left(u_{1}-u_{3}\right)+c_{2}\left(\frac{d u_{2}}{d t}-\frac{d u_{3}}{d t}\right)+c_{3}\left(\frac{d u_{1}}{d t}-\frac{d u_{3}}{d t}\right) \\
& m_{1} \frac{d^{2} u_{1}}{d t^{2}}=F+k_{3}\left(u_{3}-u_{1}\right)+k_{5}\left(u_{5}-u_{1}\right)+c_{3}\left(\frac{d u_{3}}{d t}-\frac{d u_{1}}{d t}\right)+c_{5}\left(\frac{d u_{5}}{d t}-\frac{d u_{1}}{d t}\right) \\
& m_{4} \frac{d^{2} u_{4}}{d t^{2}}=k_{4}\left(u_{5}-u_{4}\right)+c_{4}\left(\frac{d u_{5}}{d t}-\frac{d u_{4}}{d t}\right) \\
& m_{5} \frac{d^{2} u_{5}}{d t^{2}}=k_{5}\left(u_{1}-u_{5}\right)+k_{4}\left(u_{4}-u_{5}\right)+c_{5}\left(\frac{d u_{1}}{d t}-\frac{d u_{5}}{d t}\right)+c_{4}\left(\frac{d u_{4}}{d t}-\frac{d u_{5}}{d t}\right)
\end{aligned}
$$


Based on Eq. (12), the following Eq. (13) can be calculated.

$$
\left[\begin{array}{lll}
A_{11} & A_{12} & A_{13} \\
A_{21} & A_{22} & A_{23} \\
A_{31} & A_{32} & A_{33} \\
A_{41} & A_{42} & A_{43} \\
A_{51} & A_{52} & A_{53}
\end{array}\right]\left\{\begin{array}{c}
\omega^{2} \\
\omega \\
1
\end{array}\right\}=\left\{\begin{array}{l}
0 \\
0 \\
0 \\
0 \\
0
\end{array}\right\}
$$

in which

$$
\begin{aligned}
& A_{11}=m_{2} \hat{u}_{2}, A_{21}=m_{3} \hat{u}_{3}, A_{31}=m_{1} \hat{u}_{1}, A_{41}=m_{4} \hat{u}_{4}, A_{51}=m_{5} \hat{u}_{5}, A_{12}=-i c_{2}\left(\hat{u}_{3}-\hat{u}_{2}\right), \\
& A_{22}=-i\left[c_{3}\left(\hat{u}_{1}-\hat{u}_{3}\right)+c_{2}\left(\hat{u}_{2}-\hat{u}_{3}\right)\right], A_{32}=-i\left[c_{3}\left(\hat{u}_{3}-\hat{u}_{1}\right)+c_{5}\left(\hat{u}_{5}-\hat{u}_{1}\right)\right], A_{42}=-i c_{4}\left(\hat{u}_{5}-\hat{u}_{4}\right), \\
& A_{52}=-i\left[c_{4}\left(\hat{u}_{4}-\hat{u}_{5}\right)+c_{5}\left(\hat{u}_{1}-\hat{u}_{5}\right)\right], A_{13}=k_{2}\left(\hat{u}_{3}-\hat{u}_{2}\right), A_{23}=k_{3}\left(\hat{u}_{1}-\hat{u}_{3}\right)+k_{2}\left(\hat{u}_{2}-\hat{u}_{3}\right), \\
& A_{33}=k_{3}\left(\hat{u}_{3}-\hat{u}_{1}\right)+k_{5}\left(\hat{u}_{5}-\hat{u}_{1}\right)+\hat{F}, A_{43}=k_{4}\left(\hat{u}_{5}-\hat{u}_{4}\right), A_{33}=k_{4}\left(\hat{u}_{4}-\hat{u}_{5}\right)+k_{5}\left(\hat{u}_{1}-\hat{u}_{5}\right),
\end{aligned}
$$

$\hat{u}_{i}$ is the amplitude of $u_{i}$ and $\hat{F}$ is the amplitude of $F$.

In order to reveal the working mechanism, the dissipative multi-resonator microstructure is equivalent to two effective media, the effective mass and the effective metadamping. Based on Newton's second law, the following equation $F=m_{e f f} \frac{d^{2} u_{1}}{d t^{2}}+c_{e f f} \frac{d u_{1}}{d t}$ must be satisfied for this microstructure, where $c_{e f f}$ is the effective metadamping coefficient and $m_{\text {eff }}$ is the effective mass. Large $c_{\text {eff }}$ means that the damping effect is obvious, and the impact energy can be effectively dissipated. Then, the following Eq. (14) can be derived.

$$
\hat{F}=-\hat{u}_{1}\left(m_{e f f} \omega^{2}+i c_{e f f} \omega\right)
$$




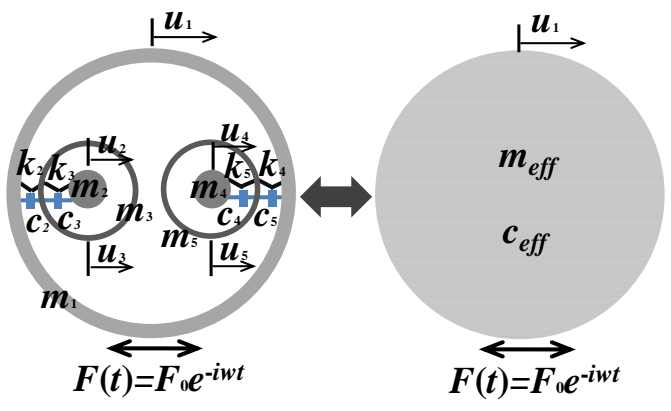

(a)

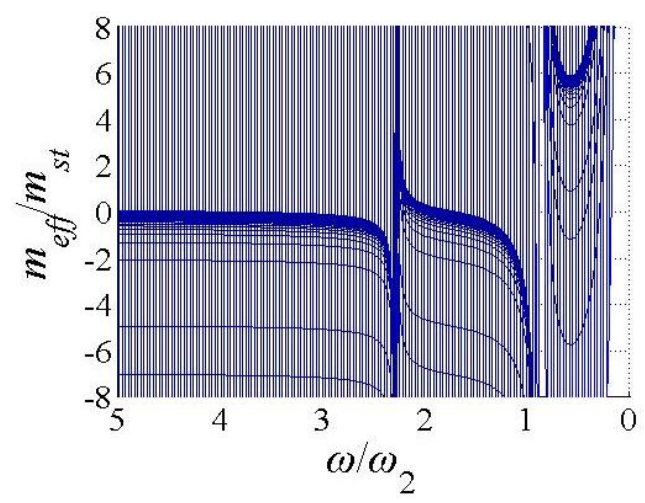

(c)

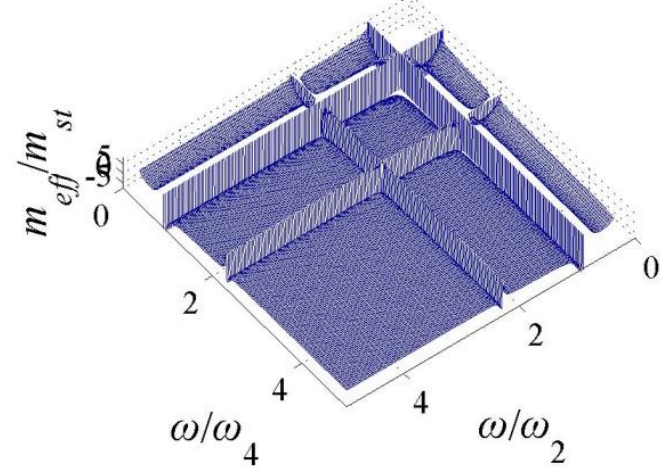

(b)

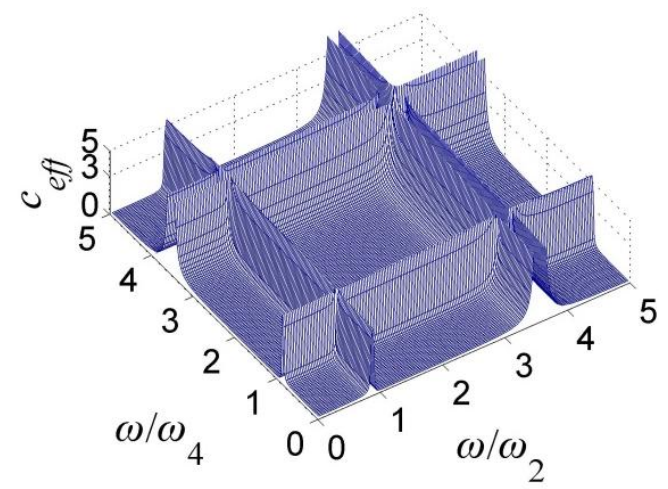

(d)

Fig. 6. (a) The dissipative multi-resonator microstructure. (b) Surface graph of $m_{e f f} / m_{s t}$ against $\omega / \omega_{2}$ and $\omega / \omega_{4}$ in global view. (c) Surface plot of $m_{\text {eff }} / m_{s t}$ against $\omega / \omega_{2}$ and $\omega / \omega_{4}$ in left view. (d) Surface graph of $c_{\text {eff }}$ against $\omega / \omega_{2}$ and $\omega / \omega_{4}$.

Figs. 6(b), (c) and (d) are the surface graphs of $m_{\text {eff }} / m_{s t}$ and $c_{\text {eff }}$ against $\omega / \omega_{2}$ and $\omega / \omega_{4}$. There are four perpendicular band gaps for $m_{e f f} / m_{s t}$ in Fig. 6(b) which is the same as the multi-resonator microstructure. Fig. 6(c) presents the left view of $m_{e f f} / m_{s t}$ surface graph. It is clear that there are two band gaps on the $\omega / \omega_{2}$ axis. Fig. $6(d)$ shows four band gaps for the metadamping surface graph, and the value of effective metadamping $c_{\text {eff }}$ is always positive. Its magnitude is significantly affected by the resonant frequencies. Near first and second resonant frequencies on the $\omega / \omega_{2}$ and $\omega / \omega_{4}$ axes, the value of $c_{\text {eff }}$ is significantly improved. Therefore, the mechanical wave can be obviously dissipated at the resonance frequencies with the DMM.

\subsection{Impact analyses of dissipative multi-resonator model (DDM)}

The sandwich panel structure with dissipative multi-resonator is shown in Fig. 7. In this model, the damping coefficients are $c_{1}=1.0 \mathrm{Nm} / \mathrm{s}, c_{2}=5.0 \mathrm{Nm} / \mathrm{s}, c_{3}=7.0 \mathrm{Nm} / \mathrm{s}$ 
and $c_{4}=2.0 \mathrm{Nm} / \mathrm{s}$. The impact results are shown in Fig. 8 and Table 3. In Fig. 8(a) and Table 3, the peak impact force of the DMM is $14.01 \mathrm{kN}$, which is obviously attenuated compared with the original and MM. Furthermore, the kinetic energy is significantly reduced and the internal energy is obviously raised from Fig. 8(b). The oscillation frequency and amplitude of impact force and impact energy of DMM are reduced quickly over time compared with the original and MM. The values of internal energy at $20 \mathrm{~ms}$ (INT20) and kinetic energy at $20 \mathrm{~ms}$ (KIN20) are $30.67 \mathrm{~J}$ and $3.08 \mathrm{~J}$. It is obvious that the impact energy is significantly dissipated. All of these results obviously demonstrate the attenuation and dissipation effects of DMM on the stress waves. The combination of viscoelastic and oscillators improves the performances of the panel structure.

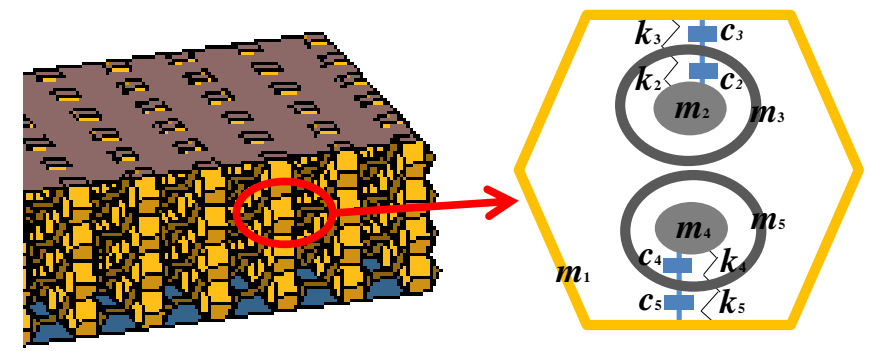

Panel structure

Dissipative multi-resonator microstructure

Fig. 7. The panel structure with dissipative multi-resonators.

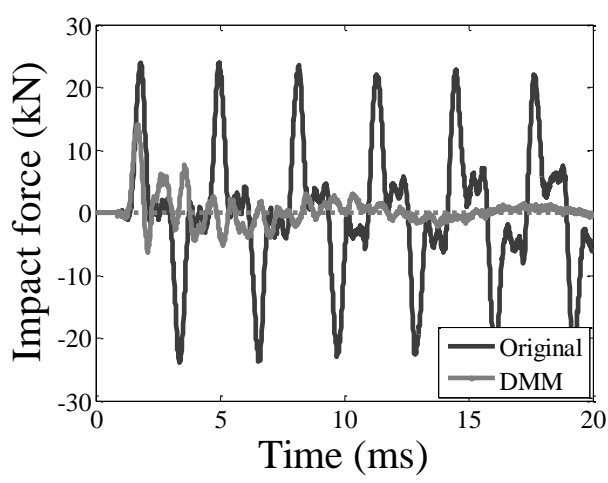

(a)

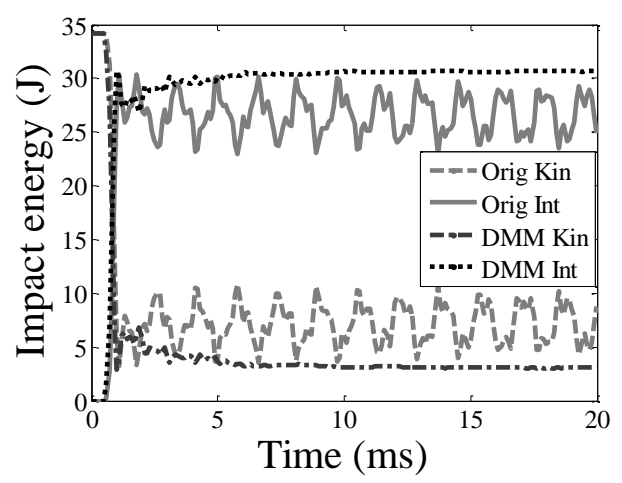

(b)

Fig. 8. Plots of (a) the impact force curve, (b) the internal and kinetic curves of DMM.

Table 3. The impact results of DMM.

\begin{tabular}{cccc}
\hline & Peak impact force $(\mathrm{kN})$ & INT20 $(\mathrm{J})$ & KIN20 $(\mathrm{J})$ \\
\hline DMM & 14.01 & 3.08 & 30.65 \\
\hline
\end{tabular}




\section{Optimize the DMM}

It is clear that the spring stiffness, oscillator masses and damping coefficients significantly affect the performance of DMM. Especially for the oscillator mass, heavier resonator can absorb and store more impact energy. However, it is worth noting that heavy oscillator increase the structure mass and restrict the application of DMM. Therefore, the parameters of DMM should be designed to improve the structural response and reduce the structure mass. Then, several optimization problems are proposed, and the optimization goals include the maximization of force attenuation and energy dissipation as well as the minimization of structure mass and unit cell number. The parameters of spring stiffness, oscillator mass and damping coefficient are set as the design variables. The entire optimization process is split into two steps to reduce the design variables in one optimization process. In addition, due to the symmetry of the structure, one quarter of the entire plate structure is taken as the design area. Among these parameters, the spring stiffness and the resonator mass should be optimized together, as they affect the resonance frequencies and band gaps together. First, regarding the parameters of spring stiffness and resonator mass in all unit cells of the plate structure as the design variables, and the damping coefficients remain constant. The NSGA-II is applied as the optimization theory. The optimization goals are to maximize the load attenuation and minimize the structure mass. After this optimization process, the light unit cells are removed to reduce the number of cells. Finally, with these designed unit cells, the damping coefficients are optimized. In this process, the goal is to minimize the peak impact force, and AT algorithm is applied to deal with this single-object problem.

\subsection{Optimize the spring stiffness and the oscillator mass}

The optimization analysis is carried out with the spring stiffness and the resonator mass. The design spaces of the variables are shown in Table 4 . There are twenty-four groups of unit cells in this model, and each group has four stiffness and four mass parameters. In order to further simplify the optimization process, the 
relationship of the spring stiffness and vibrator mass are defined as $k_{3}=3.3 k_{2}=2.4 k_{4}=$ $0.7 k_{5}$ and $m_{2}=m_{3}=m_{4}=m_{5}$, and the total design variables are reduced to forty-eight. The mathematical model of the multi-objective optimization problem is written as Eq. (15).

$$
\left\{\begin{array}{l}
\text { Min } F_{\text {peak }}, M \\
\text { s.t. } \\
0.0002 \mathrm{~N} / \mathrm{mm} \leq k_{3} \leq 0.1 \mathrm{~N} / \mathrm{mm} \\
0.0 \mathrm{~kg} \leq m_{2} \leq 0.008 \mathrm{~kg} \\
k_{3}=3.3 k_{2}=2.4 k_{4}=0.7 k_{5} \\
m_{2}=m_{3}=m_{4}=m_{5}
\end{array}\right.
$$

where $F_{\text {peak }}$ is the peak impact force, and $M$ is the mass of DMM. The Pareto frontier of this problem is illustrated in Fig. 9. As shown in Fig. 9, fifty feasible solutions are exhibited for us to select.

Table 4. The design space of spring stiffness $k_{3}$ and resonator mass $m_{2}$.

\begin{tabular}{ccc}
\hline Variables & Lower & Upper \\
\hline$k_{3}(\mathrm{~N} / \mathrm{mm})$ & 0.0002 & 0.1 \\
$m_{2}(\mathrm{~kg})$ & 0.0 & 0.008 \\
\hline
\end{tabular}

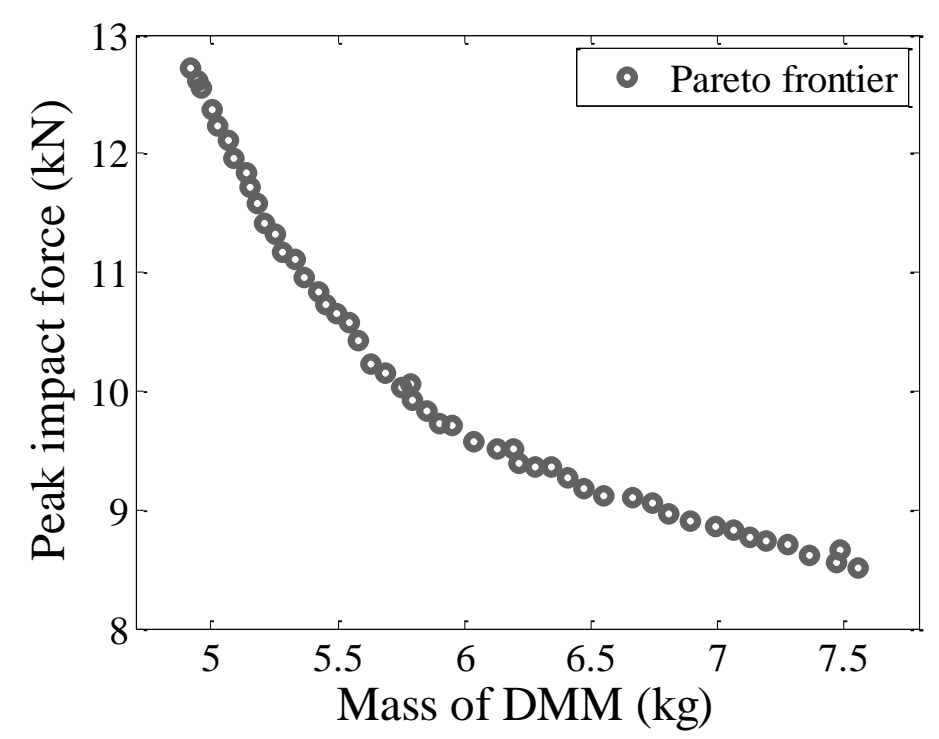

Fig. 9. The Pareto frontier solutions of the WDMM.

In this work, a small structure mass is preferred. Then, the impact responses of the optimal solution are shown in Fig. 10(a) and Table 5, and its peak force, DMM mass, INT20 and KIN20 are $10.42 \mathrm{kN}, 5.59 \mathrm{~kg}, 30.59 \mathrm{~J}$ and $3.11 \mathrm{~J}$, respectively. 
Furthermore, this optimal model is called the whole designed DMM (WDMM) for the sake of convenience. It is obvious that the impact force of the WDMM is obviously attenuated compared with the original and DMM. Meanwhile, the values of INT20 and KIN20 of WDMM are similar to those of DMM. Therefore, the impact energy is also significantly dissipated. In addition, the mass of all resonators in WDMM are reduced from $12.16 \mathrm{~kg}$ to $5.59 \mathrm{~kg}$. These results demonstrate that the optimal parameters of the DMM can effectively improve the performances of the panel structure. The distributions of $m_{2}$ and $k_{3}$ of each group in WDMM are shown in Figs. 11(a), (b) and Tables 6, 7. In Figs. 11(a) and (b), the diameter of the ball and the size of the spring are linear with the values of $m_{2}$ and $k_{3}$. Therefore, a big ball means a heavy resonator, and a big ring implies the high stiffness of the spring. The mass and spring stiffness distributions of all cells can be roughly seen by using these figures. In Fig. 11 (a), it can be seen that the resonator masses in the impact zone are bigger than those of other regions. This is because the attenuation and dissipation of stress waves requires heavy resonators.

Table 5. The crash results of all models.

\begin{tabular}{cccccc}
\hline & $\begin{array}{c}\text { Peak impact } \\
\text { force }(\mathrm{kN})\end{array}$ & INT20 $(\mathrm{J})$ & KIN20 $(\mathrm{J})$ & Mass $(\mathrm{kg})$ & $\begin{array}{c}\text { Unit cell } \\
\text { number }\end{array}$ \\
\hline Original & 23.9 & - & - & - & - \\
DMM & 14.01 & 3.08 & 30.65 & 12.16 & 608 \\
WDMM & 10.42 & 3.11 & 30.59 & 5.59 & 608 \\
RDMM & 10.84 & 3.10 & 30.60 & 5.37 & 496 \\
\hline
\end{tabular}

Table 6 . The values of designed $m_{2}$ in all groups.

\begin{tabular}{cccccc}
\hline & $m_{2}(\mathrm{~kg})$ & Group & $m_{2}(\mathrm{~kg})$ & Group & $m_{2}(\mathrm{~kg})$ \\
\hline Group 1 & 0.003118 & Group 3 & 0.000756 & Group 5 & 0.001162 \\
Group 2 & 0.000922 & Group 4 & 0.000267 & Group 6 & 0.001075 \\
Group 7 & 0.002752 & Group 9 & 0.002238 & Group 11 & 0.002139 \\
Group 8 & 0.001731 & Group 10 & 0.003148 & Group 12 & 0.001703 \\
Group 13 & 0.004082 & Group 15 & 0.003313 & Group 17 & 0.000422 \\
Group 14 & 0.000484 & Group 16 & 0.000961 & Group 18 & 0.001130 \\
Group 19 & 0.006745 & Group 21 & 0.003135 & Group 23 & 0.003883 \\
Group 20 & 0.000856 & Group 22 & 0.003902 & Group 24 & 0.007492 \\
\hline
\end{tabular}

Table 7. The values of designed $k_{3}$ in all groups. 


\begin{tabular}{cccccc}
\hline & $k_{3}(\mathrm{kN} / \mathrm{mm})$ & Group & $k_{3}(\mathrm{kN} / \mathrm{mm})$ & Group & $k_{3}(\mathrm{kN} / \mathrm{mm})$ \\
\hline Group 1 & 0.035765 & Group 3 & 0.036333 & Group 5 & 0.090197 \\
Group 2 & 0.095675 & Group 4 & 0.043323 & Group 6 & 0.077760 \\
Group 7 & 0.065625 & Group 9 & 0.054805 & Group 11 & 0.063241 \\
Group 8 & 0.012946 & Group 10 & 0.056479 & Group 12 & 0.043216 \\
Group 13 & 0.092746 & Group 15 & 0.086920 & Group 17 & 0.044580 \\
Group 14 & 0.037566 & Group 16 & 0.081782 & Group 18 & 0.001069 \\
Group 19 & 0.075173 & Group 21 & 0.010816 & Group 23 & 0.093135 \\
Group 20 & 0.084223 & Group 22 & 0.001989 & Group 24 & 0.024374 \\
\hline
\end{tabular}

From Fig. 11(a) and Table 6, there are many light unit cells, and these cells have less influence on the performances of WDMM. In addition, for practical engineering problems, more unit cells mean more design and manufacturing costs. Therefore, these unit cells with oscillator masses less than $0.0008 \mathrm{~kg}$ are deleted, and the WDMM with less unit cells is named the reduced DMM (RDMM). Finally, the number of cells of the RDMM decreases from 608 to 496, and the impact results of the RDMM are shown in Fig. 10(b) and Table 5. The peak force, structure mass, INT20 and KIN20 of the RDMM are $10.84 \mathrm{kN}, 5.37 \mathrm{~kg}, 30.60 \mathrm{~J}$ and $3.10 \mathrm{~J}$, respectively. Compared with the WDMM, the peak force of the RDMM is slightly increased, while its structure mass and unit cell number are significantly reduced. Figs. 11(c) and (d) show the distributions of $m_{2}$ and $k_{3}$ of all cells. It is clear that these light oscillators as well as their corresponding springs on the both side and the middle regions of the structure are removed.
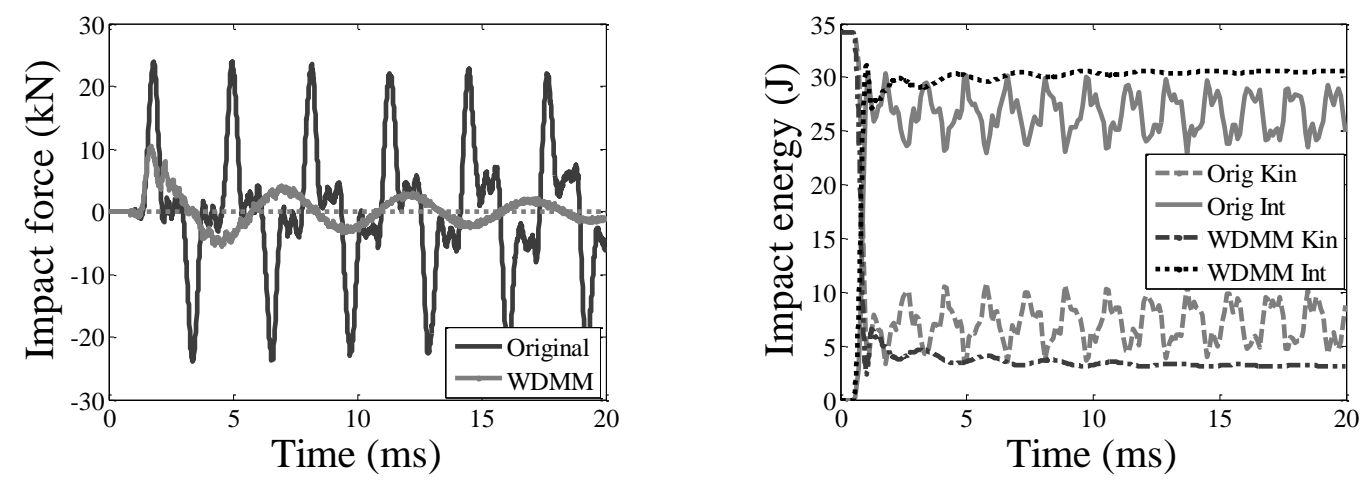

(a) 

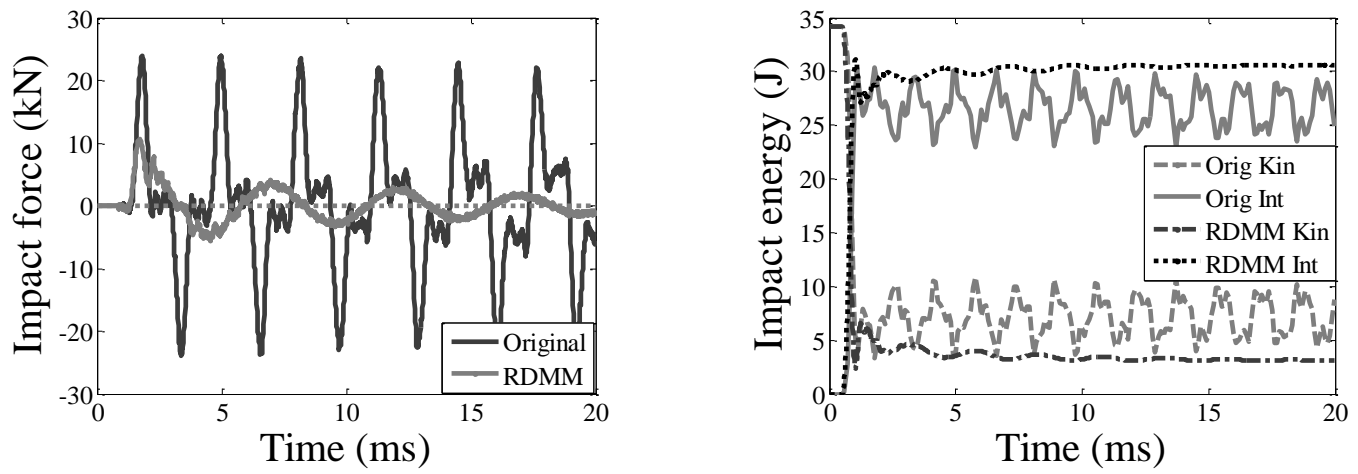

(b)

Fig. 10. Impact force and impact energy curves of (a) the WDMM, (b) the RDMM.

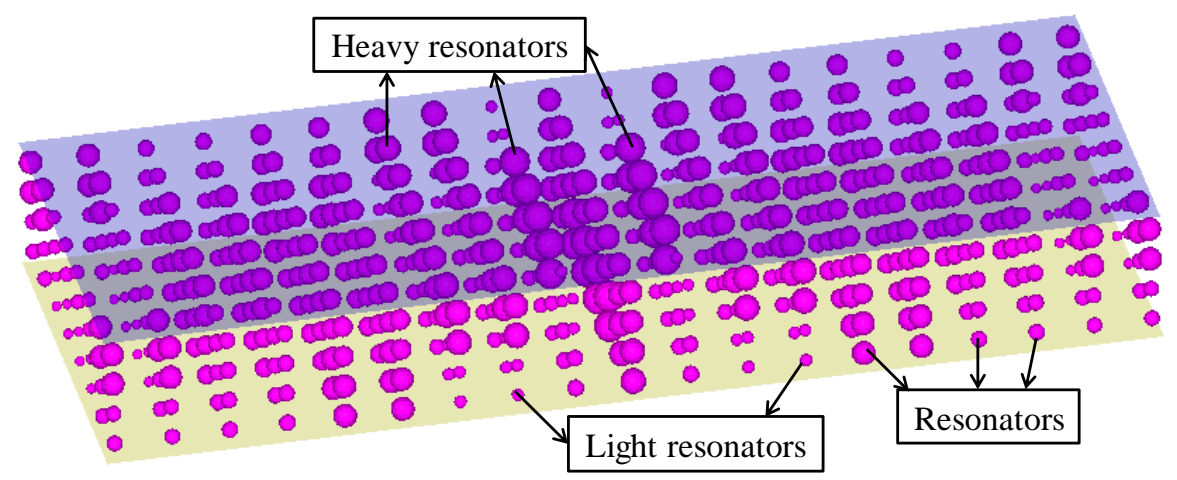

(a)

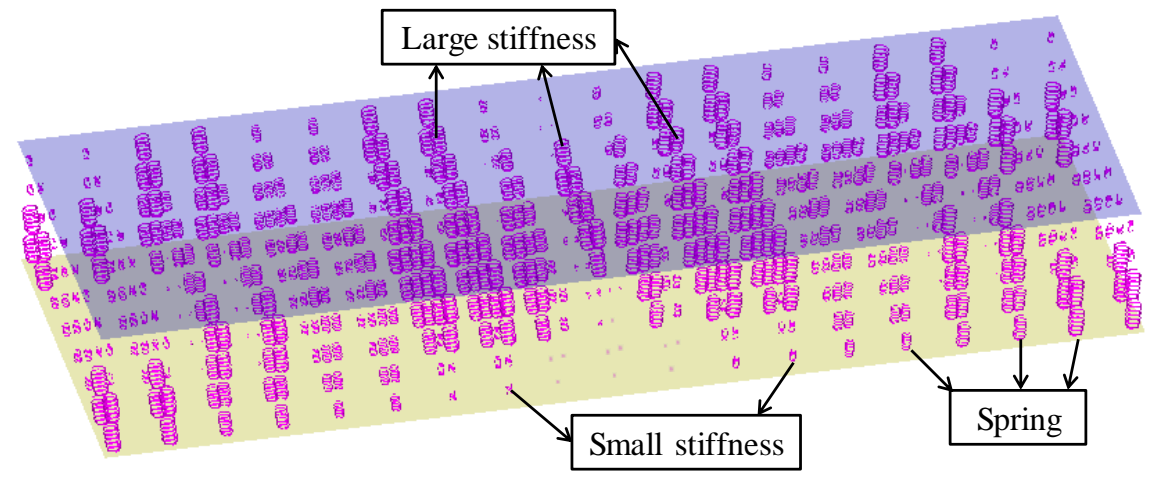

(b)

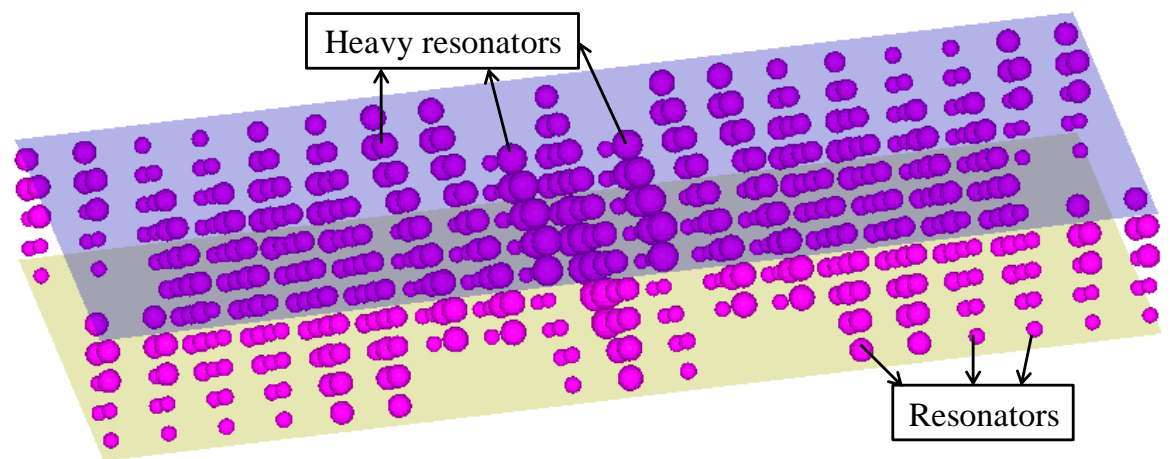

(c) 


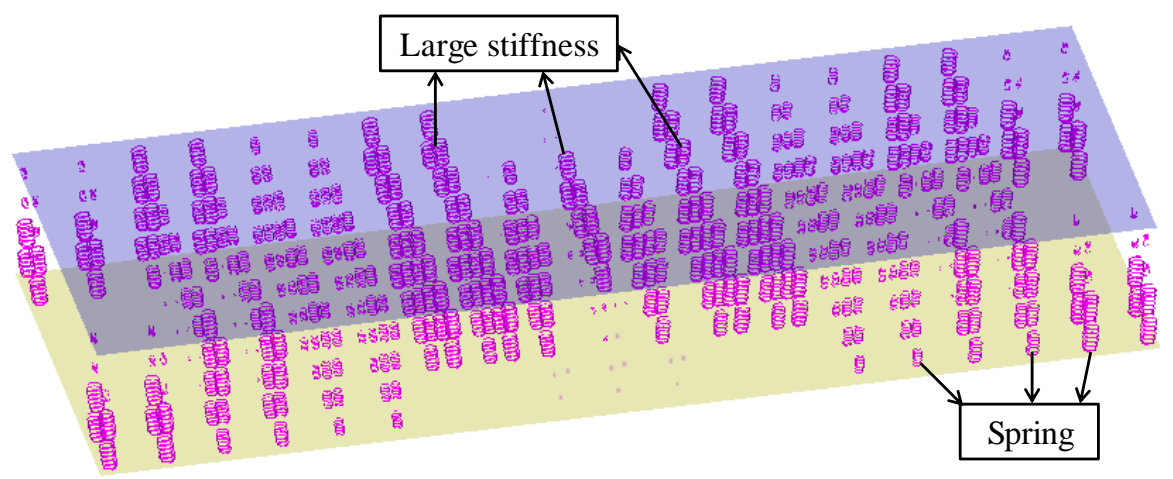

(d)

Fig. 11. The distributions of (a) resonator mass and (b) spring stiffness of the WDMM. The distributions of (c) resonator mass and (d) spring stiffness of the RDMM.

\subsection{Optimize the damping coefficients}

The optimization analysis is carried out with the damping coefficients to further enhance its attenuation and dissipation effects. This optimization model contains six groups of cells and twenty-four design variables. The goal is to minimize the impact force, and the optimization theory is AT. The range of $c_{2}$ is from $1 \mathrm{Nm} / \mathrm{s}$ to $80 \mathrm{Nm} / \mathrm{s}$. The mathematical model of this single-objective problem is shown as follows.

$$
\left\{\begin{array}{l}
\text { Min } F_{\text {peak }} \\
\text { s.t. } \\
1 \mathrm{Nm} / \mathrm{s} \leq c_{2} \leq 80 \mathrm{Nm} / \mathrm{s} \\
1 \mathrm{Nm} / \mathrm{s} \leq c_{3} \leq 80 \mathrm{Nm} / \mathrm{s} \\
1 \mathrm{Nm} / \mathrm{s} \leq c_{4} \leq 80 \mathrm{Nm} / \mathrm{s} \\
1 \mathrm{Nm} / \mathrm{s} \leq c_{5} \leq 80 \mathrm{Nm} / \mathrm{s}
\end{array}\right.
$$

The optimal model is called the designed damping RDMM (DDMM), and the optimum results are shown in Fig. 12 and Table 8. From Fig. 12(a), (b) and Table 8, the peak impact force, INT20 and KIN20 of DDMM are $9.10 \mathrm{kN}, 2.23 \mathrm{~J}$ and $31.44 \mathrm{~J}$, respectively. The optimal damping coefficients of each group are shown in Table 9. It is obvious that the values of peak impact force and KIN20 of DDMM are the smaller than those of RDMM, and its value of INT20 is the larger than that of RDMM. Therefore, the results of the DDMM are significantly better than those of the RDMM, and the mitigation of peak force and the dissipation of impact energy are very obvious. Fig. 12(c) shows the displacement nephogram of the panel structure at different time. 
It is clear that the deformation is mainly concentrated in the impact area of the structure during the entire collision process. In other zone, the structure deformation is very small. Compared with the original model, the deformation of the DDMM in other regions is significantly reduced. Therefore, the structures outside the collision region can be effectively protected with DDMM.

The greatly improved performance of the DDMM in dynamic load mitigation and impact energy dissipation indicates the wide application prospects of the combination of honeycomb sandwich structure and resonators, and this part of work can be further extend to other areas. For example, this combined structure can be applied to alleviate the impact force in vehicle collision, isolate the impact load and mechanical vibration suffered by power battery and relieve damage caused by explosion dynamic loading.

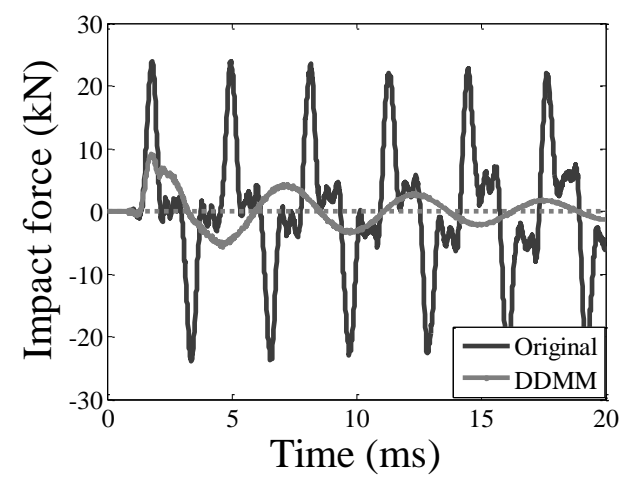

(a)

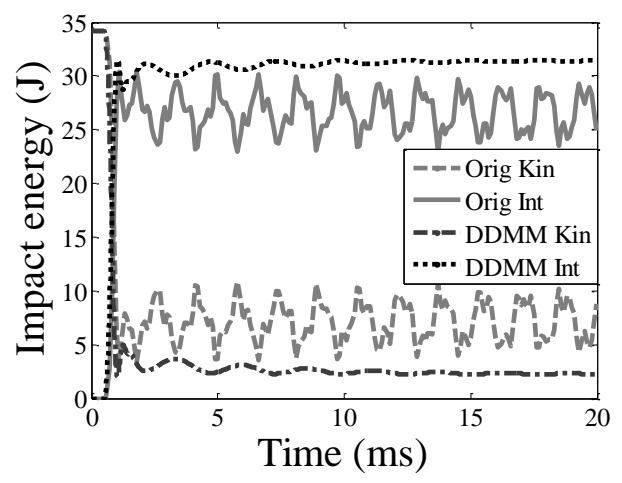

(b) 

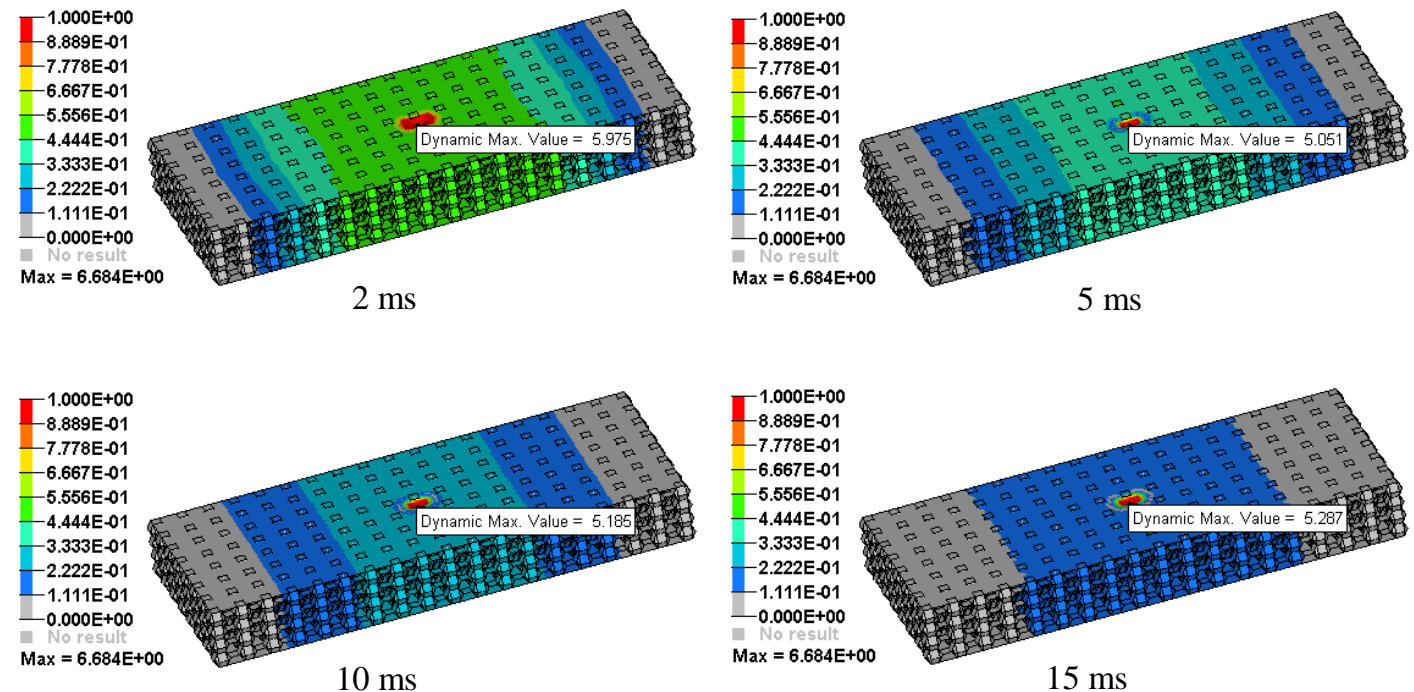

$15 \mathrm{~ms}$

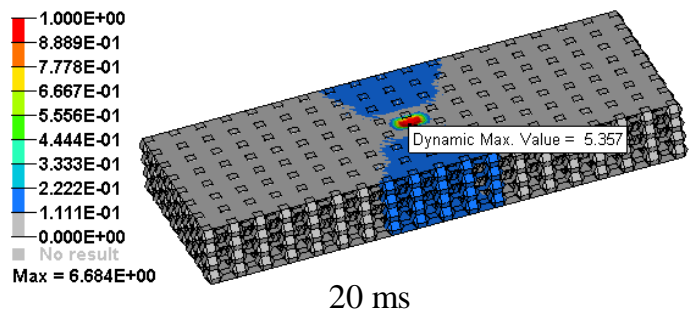

(c)

Fig. 12. (a) Impact force curve, (b) energy curves, (c) deformation nephogram of the DDMM.

Table 8. The impact results of the DDMM

\begin{tabular}{cccccc}
\hline & $\begin{array}{c}\text { Peak impact force } \\
(\mathrm{kN})\end{array}$ & $\mathrm{INT} 20(\mathrm{~J})$ & KIN20 (J) & Mass (kg) & $\begin{array}{c}\text { Unit cell } \\
\text { number }\end{array}$ \\
\hline DDMM & 9.10 & 31.44 & 2.13 & 5.37 & 496 \\
\hline
\end{tabular}

Table 9. The designed damping coefficients of each group

\begin{tabular}{ccccc}
\hline & $c_{1}(\mathrm{Nm} / \mathrm{s})$ & $c_{2}(\mathrm{Nm} / \mathrm{s})$ & $c_{3}(\mathrm{Nm} / \mathrm{s})$ & $c_{4}(\mathrm{Nm} / \mathrm{s})$ \\
\hline Group 1 & 0.02215 & 0.04018 & 0.03982 & 0.00200 \\
Group 2 & 0.00700 & 0.02954 & 0.03840 & 0.03031 \\
Group 3 & 0.00584 & 0.02567 & 0.05061 & 0.00182 \\
Group 4 & 0.00254 & 0.04165 & 0.05585 & 0.00387 \\
Group 5 & 0.02452 & 0.01196 & 0.01296 & 0.01964 \\
Group 6 & 0.05207 & 0.05837 & 0.05006 & 0.05971 \\
\hline
\end{tabular}

\section{Discussion of the practical design and manufacturing of LMs}

This section presents a summary and outlook of the practical design and manufacturing of LMs. LMs have a variety of structural forms, and previous studies 
have already given advices on the design of LMs. One kind of LMs was fabricated with the centimeter-sized lead balls and the resin coating of a thickness of $2.5 \mathrm{~mm}$ (Ping et al. 2000). The coatings and spheres are arranged in a simple cubic crystal with a lattice constant of $1.55 \mathrm{~cm}$. Two types of LMs were provided by (Manimala et al. 2014). One is the coil spring oscillator and the other is the improved flat spring oscillator. The local resonance frequency of the coil spring resonator varies greatly, and the flat spring oscillator uses a thin steel plate designed by finite element simulation to provide a more accurate stiffness for the required local resonant frequency. A spring mass system was proposed by (Khan, Li, and Tan 2018). In this system, carbon steel is used as the material of inner oscillator, and the lightweight aluminum alloy structure is used for external mass. Coil springs are used to connect internal and external masses, and adhesives with epoxy and hardener connect the springs to internal and external masses. For the current work of (Barnhart et al. 2019), the dissipative double resonator microstructure which is embedded in an epoxy resin matrix by a double layered spherical inclusion was fabricated. The experiment results show this microstructure can achieve the mitigation of broadband elastic wave.

The conceptual design to combine the honeycomb structure and resonators to mitigate the impact load and dissipate the impact energy is presented in this work. Based on the results of theoretical analyses and numerical calculations, it is seen that the impact responses of the sandwich panel structure are greatly improved. It is clear that the combination of resonators and honeycomb sandwich structures shows the great potential for structural collision problems. In addition, our work is in its infancy, and there is still much room to explore, especially in the actual design, manufacturing and testing of LMs.

\section{Conclusion}

In this paper, the extraordinary attenuation effects of a decahedral honeycomb sandwich panel structure with internal resonators are studied. Combined with the matrix structure, the resonators present the bizarre property of negative effective mass, and the combination of damping characteristic and local resonance of resonator 
exhibits the phenomenon of metadamping. The theoretical and numerical analyses show that these novel properties can effectively attenuate the impact load and dissipate the impact energy. In order to further enhance the structure responses, the spring stiffness, lumped mass and damping coefficient of internal resonators are optimized based on optimization algorithms. The optimized sandwich structure presents extraordinary load attenuation and energy absorption with a relatively small structure mass. The combination of resonators and honeycomb sandwich structure shows great potential application in many areas.

\section{Disclosure statement}

No potential conflict of interest was reported by the authors.

\section{Funding}

The Project is supported by the Foundation for Innovative Research Groups of the National Natural Science Foundation of China (Grant No. 51621004), and the Opening Project of Guangxi Key Laboratory of Automobile Components and Vehicle Technology, Guangxi University of Science and Technology (No. 2017GKLACVTKF01) and the Natural Science Foundation of Hunan Province, China (Grant No. 2017JJ3030).

\section{References}

Bacigalupo, A., G. Gnecco, M. Lepidi, and L. Gambarotta. 2016. "Optimal Design of Low-Frequency Band Gaps in Anti-Tetrachiral Lattice Meta-Materials." Composites Part B 115: 341-359.

Barnhart, M. V., X. C. Xu, Y. Y. Chen, S. Zhang, J. Z. Song, and G. L. Huang. 2019. "Experimental Demonstration of a Dissipative Multi-resonator Metmaterial for Broadband Elastic Wave Attenuation." Journal of Sound and Vibration 438: 1-12.

Biagi, R., and H. Bart-Smith. 2012. "In-Plane Column Response of Metallic Corrugated Core Sandwich Panels." International Journal of Solids \& Structures 49 (26): 3901-3914.

Bouhlel, M. A., B. Nathalie, G. R. Rommel, O. Abdelkader, and M. Joseph. 2018. "Efficient Global Optimization for High-Dimensional Constrained Problems by Using the Kriging Models Combined with the Partial Least Squares Method." Engineering Optimization 50 (12): 2038-2053.

Chen, H., X. P. Li, Y. Y. Chen, and G. L. Huang. 2017. "Wave Propagation and Absorption of Sandwich Beams Containing Interior Dissipative Multi-Resonators." Ultrasonics 76: 99-108.

Chen, Y. Y., M. V. Barnhart, J. K. Chen, G. K. Hu, C. T. Sun, and G. L. Huang. 2016. "Dissipative Elastic 
Metamaterials for Broadband Wave Mitigation at Subwavelength Scale." Composite Structures 136: 358-371.

Chen, Z. Z., Z. H. Wu, X. K. Li, G. Chen, L. Gao, X. H. Gan, G. F. Chen, and S. Z. Wang. 2019. "A Multiple-Design-Point Approach for Reliability-Based Design Optimization." Engineering Optimization 51 (5): 875-895.

Deb, K., P. Amrit, A. Sameer, and T. Meyarivan. 2002. A Fast and Elitist Multiobjective Genetic Algorithm: NSGA-II." IEEE transactions on evolutionary computation 6 (2): 182-197.

Dharmasena, K. P., H. N. G. Wadley, Z. Y. Xue, and J. W. Hutchinson. 2008. "Mechanical Response of Metallic Honeycomb Sandwich Panel Structures to High-Intensity Dynamic Loading." International Journal of Impact Engineering 35 (9): 1063-1074.

Hou, S.J., C. F. Shu, S. Y. Zhao, T. Y. Liu, H. Xu, and Q. Li. 2015. "Experimental and Numerical Studies on Multi-Layered Corrugated Sandwich Panels Under Crushing Loading." Composite Structures 126: 371-385. doi: https://doi.org/10.1016/j.compstruct.2015.02.039.

Huang, H. H., and C. T. Sun. 2009. "Wave Attenuation Mechanism in an Acoustic Metamaterial with Negative Effective Mass Density." New Journal of Physics 11 (1):013003.

Islam, M. T., and G. Newaz. 2012. "Metamaterial with Mass-Stem Array in Acoustic Cavity." Applied Physics Letters 100 (1): 011904.

Jiao, Z., Y. Cheng, H. k. Zhang, G. L. Huang, and G. Hu. 2015. "Experimental Study on Interaction Between a Positive Mass and a Negative Effective Mass Through a Mass-Spring System." Theoretical \& Applied Mechanics Letters 5 (5):196-199.

Kadlec, P., and V. Šeděnka. 2018. "Particle Swarm Optimization for Problems with Variable Number of Dimensions." Engineering Optimization 50 (3): 382-399.

Khan, M. H., B. Li, and K. T. Tan. 2018. "Impact Load Wave Transmission in Elastic Metamaterials." International Journal of Impact Engineering 118: 50-59.

Krushynska, A. O., V. G. Kouznetsova, and M. G. D. Geers. 2014. "Towards Optimal Design of Locally Resonant Acoustic Metamaterials." Journal of the Mechanics \& Physics of Solids 71 (71): 179-196.

Lai, Y., Y. Wu, P. Sheng, and Z. Q. Zhang. 2011. "Hybrid Elastic Solids." Nature Materials 10 (8): 620-624.

Li E., Z. C. He, W. Gang, and G. R. Liu. 2018. "An Efficient Algorithm to Analyze Wave Propagation in Fluid/Solid and Solid/Fluid Phononic Crystals." Computer Methods in Applied Mechanics \& Engineering 333:421-342.

Li, Q. Q., Z. C. He, E. Li, and A. G. Cheng. 2019. "Design of a Multi-Resonator Metamaterial for Mitigating Impact Force." Journal of Applied Physics 125 (3): 035104. doi: 10.1063/1.5029946.

Li, Q. Q., k. Song, Z. C. He, E. Li, A. G. Cheng, and T. Chen. 2017. "The Artificial Tree (AT) Algorithm." Engineering Applications of Artificial Intelligence 65: 99-110.

Li, Q. Q., Z. C. He, E. Li, and A. G. Cheng. 2018. "Design and Optimization of Three-Rsonator Locally Resonant Metamaterial for Impact Force Mitigation." Smart Materials and Structures 27 (9): 095015.

Liu, X. N., G. K. Hu, G. L. Huang, and C. T. Sun. 2011. "An Elastic Metamaterial with Simultaneously Negative Mass Density and Bulk Modulus." Applied Physics Letters 98 (25): 251907.

Lu, C., M. Y. Zhao, J. Liu, J. Wang, Y. Gao, X. Cui, and P. Chen. 2015. "Stress Distribution on Composite Honeycomb Sandwich Structure Suffered from Bending Load." Procedia Engineering 99:405-412. Manimala, J. M., H. H. Huang, C. T. Sun, R. Snyder, and S. Bland. 2014. "Dynamic Load Mitigation Using Negative Effective Mass Structures." Engineering Structures 80: 458-468. 
Meng, H., J. H. Wen, H. G. Zhao, and X. Wen. 2012. "Optimization of Locally Resonant Acoustic Metamaterials on Underwater Sound Absorption Characteristics." Journal of Sound \& Vibration 331 (20): 4406-4416.

Mitchell, S. J., A. Pandolfi, and M. Ortiz. 2014. "Metaconcrete: Designed Aggregates to Enhance Dynamic Performance." Journal of the Mechanics \& Physics of Solids 65 (1): 69-81.

Mitchell, S. J., A. Pandolfi, and M. Ortiz. 2015. "Investigation of Elastic Wave Transmission in a MetaconcreteSlab." Mechanics of Materials 91 (9): 295-303.

Pai, P. F., P. Hao, and S. Y. Jiang. 2014. "Acoustic Metamaterial Beams Based on Multi-Frequency Vibration Absorbers." International Journal of Mechanical Sciences 79 (1): 195-205.

Ping, S., X. X. Zhang, Z. Liu, and C. T. Chan. 2000. "Locally Resonant Sonic Materials." Science 289 (5485): 1734-1736.

Sharma, B., and C. T. Sun. 2016. "Local Resonance and Bragg Bandgaps in Sandwich Beams Containing Periodically Inserted Resonators." Journal of Sound and Vibration 364: 133-146. doi: https://doi.org/10.1016/j.jsv.2015.11.019.

Sun, G. Y., X. T. Huo, D. D. Chen, and Q. Li. 2017. "Experimental and Numerical Study on Honeycomb Sandwich Panels Under Bending and in-Panel Compression." Materials \& Design 133: 154-168. doi: https://doi.org/10.1016/j.matdes.2017.07.057.

Tan, K. T., H. H. Huang, and C. T. Sun. 2012. "Optimizing the Band Gap of Effective Mass Negativity in Acoustic Metamaterials." Applied Physics Letters 101 (24): 241902.

Thompson, D. J. 2008. "A Continuous Damped Vibration Absorber to Reduce Broad-Band Wave Propagation in Beams." Journal of Sound \& Vibration 311 (3): 824-842.

Wang, C. Y., Z. L. Liu, L. J. Gao, D. D. Xu, and Z. Zhuang. 2017. "Analytical and Numerical Modeling on Resonant Response of Particles in Polymer Matrix Under Blast Wave." Computational Materials Science 140: 70-81.

Wu, J. E., X. Y. Wang, B. Tang, Z. J. He, and H. Zhao. 2017. "Elastic Metamaterial with Simultaneously Negative Mass Density, Bulk Modulus and Shear Modulus." AIP Advances 7(10): 105309.

Wu, Y. H., Q. Liu, J. Fu, Q. Li, and D. Hui. 2017. "Dynamic Crash Responses of Bio-inspired Aluminum Honeycomb Sandwich Structures with CFRP Panels." Composites Part B Engineering 121: $122-133$

Xiao, X., Z. C. He, E. Li, and A. G. Cheng. 2019. "Design Multi-Stopband Laminate Acoustic Metamaterials for Structural-Acoustic Coupled System." Mechanical Systems \& Signal Processing 115: 418-33

Xu, X. M., Y. P. Jiang, and H. P. Lee. 2017. "Multi-Objective Optimal Design of Sandwich Panels Using a Genetic Algorithm." Engineering Optimization 49 (10): 1-20.

Yang, X. F., J. X. Ma, Y. L. Shi, Y. X. Sun, and J. L. Yang. 2017. "Crashworthiness Investigation of the Bio-inspired Bi-directionally Corrugated Core Sandwich Panel Under Quasi-Static Crushing Load." Materials \& Design 135: 275-90.

Ying, W., Y. Lai, and Z. Q. Zhang. 2011. "Elastic Metamaterials with Simultaneously Negative Effective Shear Modulus and Mass Density." Physical Review Letters 107 (10): 105506. 